\title{
Caractérisation des surfaces par réflexion rasante de rayons $X$. Application à l'étude du polissage de quelques verres silicates
}

\author{
L. Névot et P. Croce \\ Institut d'Optique (*), Centre Universitaire d'Orsay, Bât. 503, BP 43, 91406 Orsay Cedex, France
}

(Reçu le $1^{\mathrm{er}}$ août 1979, révisé le 16 novembre 1979, accepté le 20 novembre 1979)

\begin{abstract}
Résumé. - La théorie présentée permet d'obtenir une formulation explicite de l'influence des rugosités ainsi que des variations locales de constante diélectrique $n^{2}$ (dues par exemple à une modification de composition ou de compacité) sur la réflexion rasante d'un faisceau de rayons $\mathbf{X}$ monochromatique, dans la mesure où les rugosités relèvent d'une distribution gaussienne et à condition que $n^{2}$ ne dépende que de la profondeur $Z$ par rapport au plan moyen de la surface éclairée.

L'analyse des verres silicatés polis mécaniquement sur polissoir en poix, à l'aide de suspensions aqueuses d'oxydes divers, révèle que la couche de polissage se compose en réalité de deux zones bien distinctes. La première, tout à fait superficielle, l'épaisseur ne dépassant pas quelques dizaines d'angströms, présente une densité toujours inférieure à celle du cœur de l'échantillon et semble imputable au fluage plastique et à l'hydrolyse de la surface pendant le polissage. La seconde, sous-jacente, s'étend au contraire sur plusieurs centaines d'angströms et met en jeu un processus soit de densification (silice pure, alumino-silicate) soit de lacunisation (verres à assez forte teneur en ions alcalins).

Nous examinons également l'influence de la durée du polissage, du type d'oxyde utilisé, et (ou) des traitements thermiques effectués après polissage, sur les divers paramètres qui caractérisent ces couches.
\end{abstract}

\begin{abstract}
A theory is elaborated in order to explain the effects of surface roughness and locally varying bulk dielectric constant $n^{2}$ (due to changes either in composition or in compaction of the material) upon grazing X-ray reflection. An explicit formulation is derived assuming that surface heights have a gaussian distribution and $n^{2}$ is only related to depth as measured from the mean plane of the irradiated surface.

Analysis of silicate glasses mechanically polished on a pitch tool, with various oxides suspended in water, shows that the polish layer always consists in two well separated zones. The first one is the outer layer, non exceeding a few nanometers in thickness, has a density lower than of the bulk and might be due to both plastic creeping and hydrolysis during the polishing process. The second is the underlying layer which extends up to tens of nanometers and results either from a densification process, as in pure silica or alumino silicate glasses, or from a depletion process as in high alkaline silicate glasses.

The effects of time in polishing and of the oxides used as well as later heat treatments are examined in correlation with the parameters characterizing both layers.
\end{abstract}

Introduction. - Bien que le polissage du verre soit pratiqué depuis fort longtemps, et que l'on sache réaliser aujourd'hui des surfaces de très faible rugosité (possibilité largement exploitée dans l'optique instrumentale moderne), il n'en demeure pas moins que le mécanisme même du polissage n'est pas complètement élucidé. Parmi la très vaste littérature consacrée à ce problème, trois courants d'idée principaux semblent se dégager :

a) érosion moléculaire mécanique de la surface (Lord Rayleigh [1]);

(*) Laboratoire associé au C.N.R.S. b) fluage thermique superficiel (G. T. Beilby [2], W. Klemm et A. G. Smekal [3], E. Brüche et H. Poppa [4]);

c) formation d'une couche hydrolysée et érosion chimique de la surface (I. Grebenščikov [5], A. Kaller [6], M. Bourgeaux et I. Peyches [7]).

Si l'on admet en général la formation d'une couche superficielle, les diverses expériences réalisées jusqu'ici, pour déterminer la nature de cette couche (composition, compacité, etc...) et son épaisseur conduisent à des résultats très dispersés, parfois contradictoires, ceci pouvant fort bien résulter de la très grande variété de composition des verres étudiés et de la diversité des techniques de polissage employées. 
On notera aussi que, dans les études antérieures, il n'est pas tenu compte de l'influence éventuelle de la rugosité de surface pour l'interprétation fine des résultats, cette rugosité étant déterminée en général par une technique d'analyse tout autre (voir article de revue de Jean M. Bennett [8]).

Comme nous le montrerons ici, l'analyse par réflexion rasante des rayons $\mathrm{X}$, permet d'obtenir l'ensemble de ces données, et ceci de manière non destructive, ce qui en fait une technique extrêmement précieuse pour la caractérisation des états de surface dès lors que la planéité est suffisante.

1. Historique. - Grâce au développement récent des techniques d'analyse par spectroscopie d'électrons ou d'ions (notamment A.E.S., ESCA, S.I.M.S.) de nombreux chercheurs ont pu mettre en évidence une différence de composition chimique entre la surface et la masse du verre (voir, par exemple, les articles de synthèse de J. P. Rynd et $A$. K. Rastogi [9] et de G. Onoda, D. Dove et C. Pantano [10]). Toutefois la plupart des études portent sur des verres bruts de fabrication, et fort peu, considèrent des surfaces polies mécaniquement. Nous citerons Paula Heyndryckx [11] qui examine des glaces polies soit à l'oxyde de cérium, soit à l'oxyde de fer en suspension aqueuse, et C. G. Pantano et al. [12] pour des bio-glass polis à sec.

Les profils de concentration de $\mathrm{Na}$ et $\mathrm{Ca}$ pour ces verres polis ressemblent, du moins qualitativement, à ceux trouvés sur des verres non surfacés (verres flottés, soufflés, ou polis au feu) ce qui conforte l'existence d'une couche superficielle appauvrie en sodium et en calcium sur une profondeur de quelques centaines à quelques milliers d'angströms.

Pour enregistrer ces profils il est nécessaire de procéder, préalablement ou simultanément à l'analyse, à un décapage progressif de la surface par bombardement d'ions (argon le plus souvent), ce qui n'est pas sans soulever certaines complications, d'une part pour l'interprétation finale des spectres (possibilité de ségrégation au cours de la pulvérisation, de diffusion ou migration de certains constituants sous l'action de gradients thermiques ou électriques) d'autre part pour l'étalonnage en épaisseur (conversion du temps d'érosion en épaisseur).

L'analyse à l'aide de techniques non destructives et non limitées aux toutes premières couches atomiques, apparaît donc à la fois nécessaire et complémentaire des précédentes. L'excitation de la surface et le mode de détection ne font alors appel qu'à des rayonnements électromagnétiques de longueur d'onde située soit dans le visible (ellipsométrie, diffusion optique) soit dans le domaine des rayons $\mathrm{X}$ (fluorescence $X$, réflexion rasante $X$ ).

L'utilisation de la fluorescence $X$ dans le cas des verres soulève un certain nombre de difficultés liées d'une part à la nécessité d'effectuer sous vide la détection des raies d'émission $K$ de la plupart des atomes en présence (lesquels sont presque tous de numéro atomique faible, en particulier : bore, oxygène, sodium et silicium) et d'autre part à la forte pénétration du rayonnement excitateur. Dans le cas des rayons $\mathrm{X}$ moyens (longueur d'onde de $1 \AA$ environ) la profondeur utile, ou intégrée, reste de l'ordre du micron même lorsque l'attaque s'effectue de façon très rasante $(P$. Croce, $L$. Névot [13]). L'analyse par fluorescence $\mathrm{X}$ est donc avant tout qualitative. Cependant elle a permis à C. Legrand et al. [14] de constater un très léger enrichissement en silice $(4 \%)$ de la surface d'un verre à glace à la suite de son polissage à l'oxyde de fer. Notons que dans un avenir assez proche, les sources très puissantes de rayons $X$ mous que sont les synchrotrons devraient redonner un certain intérêt pour la fluorescence X. L'absorption moyenne augmentant lorsque la longueur d'onde du rayonnement excitateur croît, la profondeur utile diminue d'autant, ce qui améliore la sensibilité de la méthode. D'autre part la possibilité de travailler de part et d'autre du bord d'absorption $K$ d'un élément donné, devrait permettre un dosage plus précis de cet élément.

Les autres méthodes, citées précédemment, ne font intervenir que la composition moyenne du volume éclairé par l'intermédiaire de l'indice de réfraction.

Ainsi, grâce à des mesures ellipsométriques, $\mathbf{H}$. Yokota et al. [15-16] de même que H. Sakata [17] constatent que l'indice de réfraction en surface $n_{\mathrm{f}}$ diffère de celui de la masse $n_{\mathrm{b}}$ pour un grand nombre de verres optiques polis à l'oxyde de cérium. Dans le cas de la silice pure, du Vycor et du Pyrex, $n_{\mathrm{f}}$ est supérieur à $n_{\mathrm{b}}$. Par contre pour les verres de type crown et flint, $n_{\mathrm{f}}$ devient inférieur à $n_{\mathrm{b}}$.

Le fait que la couche de polissage soit plus dense que le cœur de l'échantillon pour les verres inaltérables et au contraire moins dense pour les verres très sensibles, à l'humidité de l'air par exemple, suggère aux auteurs l'existence de deux processus antagonistes au cours du polissage : la densification sous l'action des fortes pressions exercées localement par les grains d'oxyde et l'extraction de certains ions métalliques au voisinage de la surface (action lixiviante de l'eau), ce second processus ne se manifestant pas dans le cas des verres inaltérables et devenant prépondérant pour les autres.

Précisons que l'analyse théorique des données expérimentales précédentes suppose à priori l'homogénéité et l'isotropie de la couche de polissage. Celle-ci est donc caractérisée par un indice de réfraction constant et une épaisseur uniforme. D'autre part, il n'est pas tenu compte des rugosités de surface. Les épaisseurs ainsi déterminées pour la couche superficielle sont de l'ordre de quelques centaines d'angströms.

P. Croce et L. Prod'homme [18] analysant la diffusion optique dans le visible par des surfaces de verres polis, montrent que si ces surfaces sont éclairées sous une incidence suffisamment éloignée de la 
normale, l'intensité diffusée pour la polarisation $p$ doit s'annuler dans une certaine direction appelée pseudo-brewstérienne. Ce fait, déjà prédit par Rayleigh [19], est confirmé par les indicatrices de diffusion expérimentales, mais le minimum se situe en général dans une direction différente de celle prévue théoriquement sur la base des valeurs massiques de l'indice de réfraction des verres étudiés. Le décalage est très net pour les silicates (crown et flint). Les auteurs concluent à la présence d'une couche superficielle caractérisée par un indice de réfraction limite inférieur à celui de la masse. Par ailleurs cet indice en surface apparaît très proche de celui de la silice pure. La méthode permet en outre de déduire l'allure du spectre des rugosités de surface pour les fréquences spatiales de l'ordre de $1 \mu^{-1}$. Par contre, il n'est pas possible de déterminer la profondeur affectée par le polissage.

A la suite d'analyse de couches minces métalliques déposées sur verre, par réflexion rasante de rayons $\mathrm{X}$, quelques chercheurs ont été amenés à examiner de plus près la réflexion produite par le verre nu. Ainsi dès 1957, Nancy J. Scott (voir W. Petzold [20]) évoque l'existence d'une couche superficielle d'épaisseur égale à $50 \AA$ et de densité inférieure de $20 \%$ à celle de la masse pour un verre tendre. Rappelons que cette technique consiste à étudier la variation de l'intensité $I \mathrm{du}$ faisceau réfléchi spéculairement en fonction de l'angle d'attaque $\theta$ du faisceau incident (défini ici par rapport au plan moyen de la surface éclairée). La simulation de la courbe expérimentale $I \rightarrow f(\theta)$ est tentée en appliquant les équations de Fresnel au cas d'un seul dioptre air-verre, ou au système de deux dioptres air-couche-verre massique, ces dioptres étant supposés parfaitement plans et homogènes, comme pour les analyses par ellipsométrie citées plus haut.

Notons aussi que pour les rayons $\mathrm{X}$ moyens, l'indice de réfraction $n$ est souvent écrit sous la forme

$$
n=1-\delta+i \beta \text {. }
$$

$\delta$ est lié directement à la densité électronique moyenne du matériau considéré :

$$
\delta=2,7019 \times 10^{10} \lambda^{2}\left(\rho \frac{Z^{*}}{A}\right)
$$

(unités C.G.S.). Dans cette expression $\rho$ désigne la masse volumique du matériau, $\boldsymbol{A}$ sa masse atomique, $Z^{*}$ étant pratiquement égal au nombre d'électrons par atome, au terme correctif de dispersion anomale près (Don T. Cromer, D. Liberman [21]). $\beta$ est lié, quant à lui, au coefficient d'absorption massique $\mu / \rho$ du matériau pour le rayonnement considéré, de longueur d'onde $\lambda$. Il est à remarquer que pour les atomes de numéro atomique faible, $Z * / A$ reste pratiquement égal à 0,5 . Par conséquent, même pour des compositions très variées, on retrouve 0,5 comme valeur massique de $Z^{*} / A$ pour la plupart des verres étudiés (notamment les silicates), dans la mesure où leur teneur en atomes lourds $(\mathrm{Pb}, \mathrm{Ba}$, etc...) reste faible. Ainsi la valeur de $\delta$ dépend essentiellement de celle de la masse volumique du verre considéré.

W. Hink et W. Petzold [22] obtiennent pour $\delta$ la valeur $6,8 \times 10^{-6}$ au lieu de $8,07 \times 10^{-6}$, valeur massique du borosilicate crown BK7 (Schott glass) pour le rayonnement $\mathrm{CuK}_{\alpha_{1}}(\lambda=1,5405 \AA)$. Selon Petzold [20] cette valeur pourrait même être abaissée à $5,30 \times 10^{-6}$. L'analyse de ce même verre est reprise par G. Kühnen [23] sur la base du modèle à deux dioptres en utilisant les indices massiques pour le cœur de l'échantillon. Une simulation correcte de la courbe expérimentale du verre nu est obtenue en prenant pour épaisseur $e_{\mathrm{c}}$ de la couche superficielle $65 \AA$ et pour indice $\delta_{c} 7,5 \times 10^{-6}$. Par contre la simulation des courbes relatives à des couches minces d'aluminium déposées sur ce même verre, le conduit à des valeurs quelque peu différentes :

$$
e_{\mathrm{c}}=105 \AA \text { et } \delta_{\mathrm{c}}=6,44 \times 10^{-6} .
$$

A titre indicatif, rappelons que Yokota et al. [16] estimaient, par ellipsométrie, à $400 \AA$ environ l'épaisseur de la couche de polissage du BK7.

Jusqu'ici les études par rayons $X$ rasants n'ont concerné que le voisinage immédiat de la limite de réflexion totale des courbes $I \rightarrow f(\theta)$.

Dès que l'on s'écarte vers les incidences plus élevées, on constate (voir Fig. 1) que les modèles à deux dioptres plans parfaits, d'une part, conduisent à des intensités nettement plus fortes que celles mesurées, l'écart ne faisant que s'accroître au fur et à mesure que $\theta$ augmente, et d'autre part, font apparaître des oscillations alors que la décroissance de l'intensité expérimentale est généralement monotone dans le cas des verres polis. De telles oscillations correspondent au phénomène d'interférence entre les faisceaux réfléchis sur les deux faces limites de la couche de polissage. Par conséquent l'absence de ces battements sur la courbe expérimentale traduit bien le fait que la couche de polissage doit être envisagée plutôt sous la forme d'une zone de transition où l'indice de réfraction doit varier de façon continue entre la valeur relative au milieu extérieur (air ou vide) et celle caractéristique de la composition massique du verre. En outre il est nécessaire de se préoccuper ici de l'influence des rugosités ou aspérités de la surface sur la réflexion et la diffusion des rayons $\mathrm{X}$ incidents.

2. Théorie. - Les éléments de base de cette nouvelle approche théorique du problème de la réflexion et de la diffusion des ondes électromagnétiques, par les surfaces et les interfaces à la fois rugueux et inhomogènes, ont déjà été exposés dans un certain nombre de publications (P. Croce, L. Névot et B. Pardo [24], P. Croce et L. Prod'homme [25], P. Croce [26 à 28]).

Elle présente surtout l'avantage de ne faire aucune distinction à priori entre les irrégularités géométriques (rugosités) et les inhomogénéités de composition ou de 


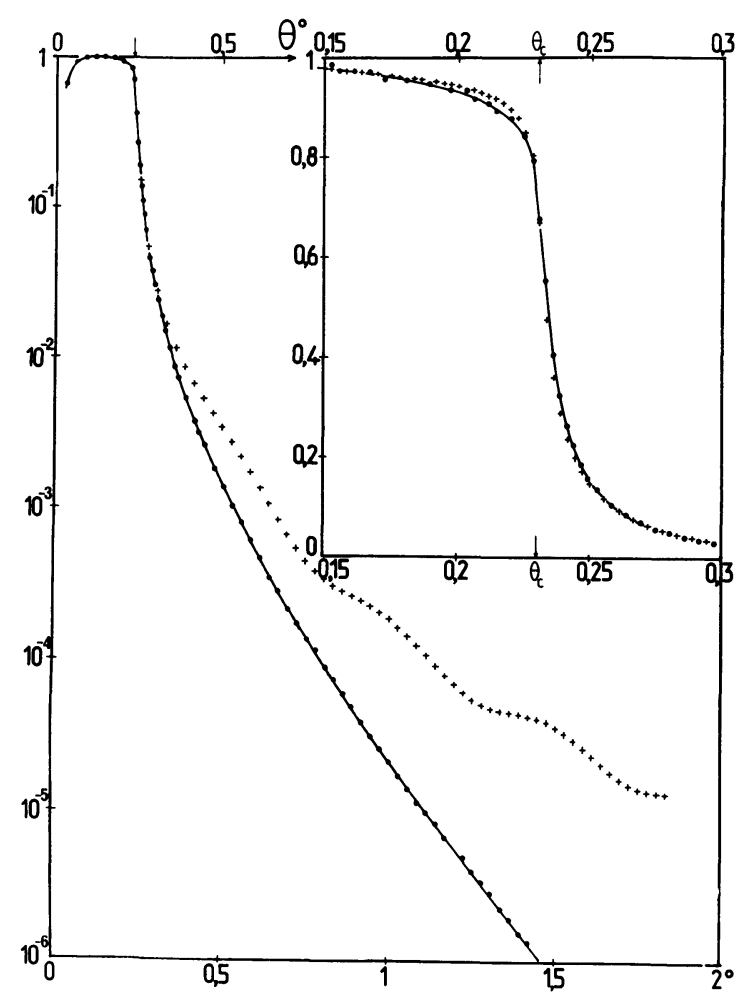

Fig. 1. - La courbe expérimentale de réflexion spéculaire $I \rightarrow f(\theta)$ reproduite en trait plein, a été obtenue sur un échantillon de borosilicate Crown B 1664 (Parra-Mantois-Sovirel) éclairé par le rayonnement $\mathrm{CuK}_{\alpha_{1}}$. Les intensités réfléchies sont données en valeurs relatives par rapport à celle mesurée dans le faisceau direct et ont été corrigées de la valeur afférente au bruit de fond. La courbe théorique, indiquée par des croix, a été tracée en admettant l'existence d'une couche superficielle de polissage, homogène et plane, d'épaisseur $90 \AA$ et de densité inférieure à celle de la masse

$$
\left[\delta_{\mathrm{c}}=7,5 \times 10^{-6} \text { au lieu de } \delta_{\mathrm{m}}=8,07 \times 10^{-6}\right] .
$$

On notera que si l'accord est acceptable pour le voisinage immédiat de la limite de réflexion totale (voir courbes pour $\theta \cong \theta_{c}$, dans la partie supérieure droite de la figure) il n'en est plus de même dès que l'on considère les angles d'incidence élevés.

[The full line shows the X-ray specular reflectance from a BSC glass surface under $\mathrm{CuK}_{\alpha_{1}}$ radiation. The reflected irradiance is referred to the direct beam and is corrected for background noise. Computed values, corresponding to an homogeneous flat polishing layer, $90 \AA$ thick and $6 \%$ less dense than the bulk, are indicated by crosses. The fair agreement near the total reflection limit falls out with increasing angle of incidence.]

compacité, celles-ci pouvant se situer soit en surface, soit à l'intérieur de l'échantillon.

Rappelons que l'on admet que le milieu réel $R$ (formé de dioptres rugueux et inhomogènes) peut être reconstitué, pour ce qui est de la répartition du champ électromagnétique, à partir du milieu idéal I (formé de dioptres plans et homogènes) à condition d'implanter dans ce dernier des sources dipolaires fictives de polarisation adéquate. $\mathrm{Si}$ au point défini par $\mathbf{r}$ règne le champ électrique $\mathbf{E}_{\mathbf{R}}(\mathbf{r})$, cette polarisation s'exprime, en unités C.G.S., par :

$$
\mathfrak{T}(\mathbf{r})=\frac{1}{4 \pi}\left[n_{\mathbf{R}}^{2}(\mathbf{r})-n_{\mathbf{I}}^{2}(\mathbf{r})\right] \mathbf{E}_{\mathbf{R}}(\mathbf{r}),
$$

$n^{2}(\mathbf{r})$ représentant la valeur de la constante diélectrique dans les milieux $R$ et $I$ respectivement.

Le problème se ramène donc à étudier le rayonnement global de ces sources en présence de I, étant précisé que de tels dipôles forcés sont supposés ne pas réagir entre eux.

Indiquons en outre que l'analyse est restreinte au seul cas des interfaces stationnaires, pour lesquels il est possible de considérer la composition dans chacun des strates découpés parallèlement au plan moyen des rugosités comme suffisamment uniforme pour que l'on puisse attribuer à ce strate un certain indice de réfraction moyen. Autrement dit nous admettons que les dimensions latérales des inhomogénéités sont suffisamment petites et que leur répartition est suffisamment régulière pour que le fait que la surface analysée varie au cours de l'expérience (elle dépend de l'angle d'attaque du faisceau incident) n'ait aucune conséquence dommageable pour l'interprétation. Ceci revient à rendre l'indice de réfraction fonction de la seule variable $Z$, distance ou cote du point considéré par rapport au plan moyen de la surface ou à tout autre plan parallèle.

\subsection{CAS D'UN INTERFACE RUGUEUX SÉPARANT} DEUX MILIEUX HOMOGÈNES. - Nous examinerons tout d'abord le cas d'un interface uniquement rugueux, les milieux situés de part et d'autre de cet interface étant homogènes et d'indices $n_{1}$ et $n_{2}$ respectivement.

Comme il a déjà été écrit dans [13] (§ 3.3.2) et dans [28] (Chap. 6), la théorie se trouve de beaucoup simplifiée si l'on peut considérer d'une part que les fréquences spatiales élevér a spectre des rugosités jouent un rôle prépondé $a ` t ~ t$ d'autre part que les indices $n_{1}$ et $n_{2}$ sont très $\mathrm{v}$ ns. Or cette dernière condition est satisfaite à mieux ue $10^{-4}$ près dans le domaine de longueurs d'onde des rayons $\mathrm{X}$ moyens. On notera toutefois que pour une discussion plus approfondie, et ceci même dans le cas d'une approximation du deuxième ordre, il serait nécessaire de connaître exactement ce spectre. Nous admettons donc que la contribution des ondes diffractées reste négligeable devant celle des ondes principales (incidente, réfléchie et transmise), même si l'amplitude des rugosités est assez forte, autrement dit même lorsque la valeur quadratique moyenne $\sqrt{\left\langle Z^{2}\right\rangle}$ n'est pas petite devant $K_{1 n}^{-1}$ et $K_{2 n}^{-1}\left(K_{1 n}\right.$ et $K_{2 n}$ désignent les composantes normales des vecteurs d'onde dans les deux milieux).

Dans les calculs antérieurs (voir P. Croce [27]) il était admis que l'on pouvait prendre pour expression des ondes réfléchie et transmise du cas réel celle des ondes homologues du cas idéal. Ici, nous chercherons une solution autocohérente, où l'amplitude de l'onde réfléchie au voisinage de l'interface pour $R$ est, par rapport à celle de $\mathrm{I}$, corrigée de l'effet de rugosité.

Les expressions générales (1) des coefficients de réflexion et de transmission en amplitude s'écrivent comme suit (voir P. Croce [28]) : 


$$
\begin{aligned}
& r_{\mathrm{R}}=r_{\mathrm{I}}+i \pi \frac{K_{0}^{2}}{K_{1 n}}\left\langle\int_{0}^{Z_{\mathrm{D}}}\left[\mathbf{E}_{\mathrm{R}}(\rho, Z) \cdot \mathbf{E}_{\mathrm{I} 1}(\rho, Z)\right]\left[n_{\mathrm{R}}^{2}(\rho, Z)-n_{\mathbf{I}}^{2}(\rho, Z)\right] \mathrm{d} Z\right\rangle_{\rho} \\
& t_{\mathrm{R}}=t_{\mathrm{I}}+i \pi \frac{K_{0}^{2}}{K_{2 n}}\left\langle\int_{0}^{Z_{\mathrm{D}}}\left[\mathbf{E}_{\mathrm{R}}(\rho, Z) \cdot \mathbf{E}_{12}(\rho, Z)\right]\left[n_{\mathrm{R}}^{2}(\rho, Z)-n_{\mathrm{I}}^{2}(\rho, Z)\right] \mathrm{d} Z\right\rangle_{\rho}
\end{aligned}
$$

$\mathbf{E}_{\mathrm{R}}(\rho, Z)$ représente le champ électrique qui existe au niveau de l'interface rugueux au point $M$ de coordonnées $\rho$ et $Z$ (voir schéma 1 , le plan de référence XOY étant pris parallèlement au plan moyen de l'interface rugueux, et l'axe $O Z$ orienté positivement

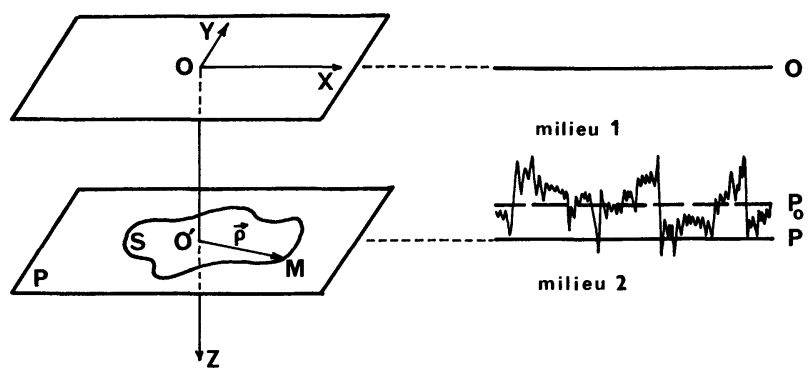

Schéma 1.

du milieu 1 vers le milieu 2) pour une onde plane incidente d'amplitude égale à 1 à l'origine $\mathrm{O}$ du repère. Le champ $E_{11}\left(E_{12}\right)$ correspond à celui qui règne en $M$ dans le cas idéal pour une onde régressive d'amplitude égale à 1 en $\mathrm{O}$, autrement dit pour une onde dont le vecteur d'onde est opposé à celui de l'onde réfléchie ou de l'onde transmise incidentes. \langle\rangle$_{\rho}$ indique que la moyenne de l'intégrale est effectuée sur une surface $\mathrm{S}$ du plan $\mathrm{P}$ suffisamment étendue pour que \langle\rangle$_{\rho}$ soit indépendant de $S$. Pour que Pexpression de $\mathbf{E}_{R}$ ne comporte que l'onde réfléchie, le plan de référence XOY doit être pris de telle sorte que les cotes $z_{D}$ des rugosités soient positives. Toutefois on pourra le faire coïncider avec le plan moyen $\mathbf{P}_{0}$ de l'interface (voir schéma 2). Ceci n'entraîne aucune modification
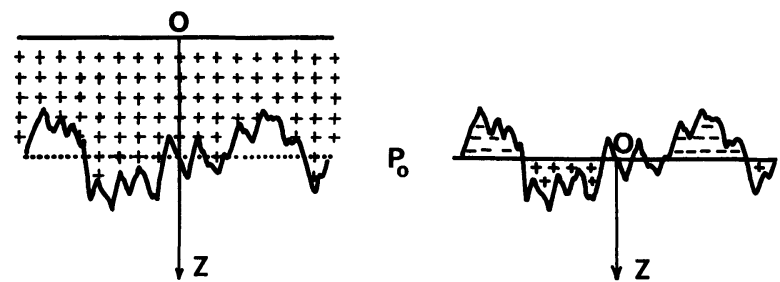

Schéma 2.

pour le calcul des intégrales de (1) pour les régions de cotes $z_{D}$ positives. Par contre dans les régions de cotes négatives il est nécessaire d'effectuer le prolongement analytique de $E_{\mathrm{R}}$ et $E_{\mathrm{l}}$ de l'autre côté de leurs dioptres respectifs, avec un signe -, lequel apparaît automatiquement du fait que $z_{D}$ change de signe.

Rappelons que dans le cas des rayons $\mathrm{X}$, les valeurs des coefficients de réflexion $r$, ou de transmission $t$, sont pratiquement les mêmes pour les polarisations « $s$ » et « $p$ ». Nous nous placerons dans le cas « $s$ » et désignerons par $\mathbf{U}_{\mathrm{Y}}$ le vecteur unitaire de l'axe $O Y$.

Les expressions de $\mathbf{E}_{\mathrm{R}}$ et $\mathbf{E}_{\mathbf{l}}$ seront par suite :

$$
\begin{aligned}
& \mathbf{E}_{\mathbf{R}} \simeq \mathrm{e}^{2 \pi K_{t} X}\left[\mathrm{e}^{2 \pi i K_{1 n} Z}+r_{\mathrm{R}} \mathrm{e}^{-2 \pi i K_{1 n} Z}\right] \mathbf{U}_{\mathbf{Y}} \\
& \mathbf{E}_{\mathrm{I}}=\mathrm{e}^{-2 \pi i K_{t} X} t_{1} \mathrm{e}^{2 \pi i K_{2 n} Z} \mathbf{U}_{\mathbf{Y}}
\end{aligned}
$$

avec :

$$
t_{1}=1+r_{1}=\frac{2 K_{1 n}}{K_{1 n}+K_{2 n}} .
$$

L'expression (1) de $r_{\mathrm{R}}$ se transforme alors comme suit (2) :

$$
\begin{aligned}
r_{\mathrm{R}} & =r_{\mathrm{I}}+\frac{i \pi}{K_{1 n}}\left(K_{1}^{2}-K_{2}^{2}\right) \frac{2 K_{1 n}}{K_{1 n}+K_{2 n}}\left\langle\int_{0}^{Z_{\mathrm{D}}} \mathrm{e}^{2 \pi i K_{2 n} Z}\left(\mathrm{e}^{2 \pi i K_{1 n} Z}+r_{\mathrm{R}} \mathrm{e}^{-2 \pi i K_{1 n} Z}\right) \mathrm{d} Z\right\rangle \\
& =r_{\mathrm{I}}+i 2 \pi\left(K_{1 n}-K_{2 n}\right)\left[\frac{\left\langle\mathrm{e}^{2 \pi i K_{1 n}+K_{2 n} Z_{\mathrm{D}}}\right\rangle-1}{2 \pi i\left(K_{1 n}+K_{2 n}\right)}+r_{\mathrm{R}} \frac{\left\langle\mathrm{e}^{2 \pi i\left(K_{2 n}-K_{1 n}\right) Z_{D}}\right\rangle-1}{2 \pi i\left(K_{2 n}-K_{1 n}\right)}\right] \\
& =r_{\mathrm{I}}+r_{\mathrm{I}}\left[\mathrm{e}^{\left.-2 \pi^{2}\left(K_{1 n}+K_{2 n}\right)^{2}<Z_{D}^{2}\right\rangle}-1\right]-r_{\mathrm{R}}\left[\mathrm{e}^{-2 \pi{ }^{2}\left(K_{1 n}-K_{2 n}\right)^{2}<Z_{D}^{2}>}-1\right] .
\end{aligned}
$$

Ce qui conduit finalement à $\left(2^{\prime}\right)$ :

$$
r_{\mathrm{R}}=r_{\mathrm{I}} \mathrm{e}^{-8 \pi^{2} K_{1 n} K_{2 n}<Z_{D}^{2}>} .
$$

La courbe théorique de réflexion spéculaire $I_{0} \rightarrow f(\theta)$ présentée en trait continu sur la figure 2 correspond à un dioptre air-verre (B 1664) parfaitement plan et homogène attaqué par le rayonnement
$\mathrm{CuK}_{\alpha 1}$. Les courbes afférentes aux symboles $\mathrm{O}$ et + sont relatives à l'expression (3) :

$$
I(\theta)=I_{0}(\theta)^{-} \exp \left[-16 \pi^{2} K_{1 n} K_{2 n}\left\langle Z_{\mathrm{D}}^{2}\right\rangle\right]
$$

la rugosité quadratique moyenne $\sigma=\sqrt{\left\langle Z^{2}\right\rangle}$ prenant les valeurs 10 et $40 \AA$ respectivement. On notera, sur cette dernière, que l'atténuation se fait déjà sentir 


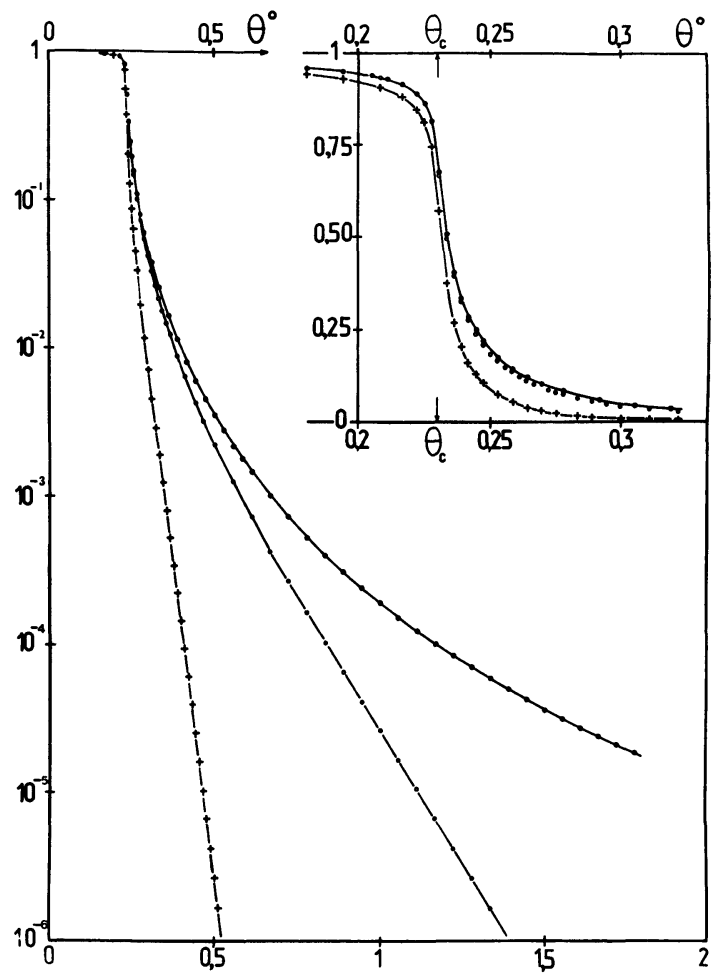

au voisinage de $\theta_{c}$, entraînant un déplacement très net de la limite de réflexion totale vers les angles $\theta$ plus rasants. On constate par ailleurs que l'expression (3) conduit à des résultats identiques à ceux fournis par le modèle de couche de passage dans laquelle l'indice de réfraction varie suivant la fonction-erreur (L. Névot, P. Croce [29]) soit :

$$
n(Z)=1+\left(n_{2}-1\right) F(Z)
$$

avec

$$
F(Z)=\frac{1}{\sigma \sqrt{2 \pi}} \int_{-\infty}^{Z} \exp -\left(\frac{z^{2}}{2 \sigma^{2}}\right) \mathrm{d} z
$$

En outre elle présente l'avantage d'une programmation plus aisée que celle de la couche de passage et requiert
Fig. 2. - Effet de la rugosité sur la courbe de réflexion spéculaire d'un borosilicate crown B 1664 supposé parfaitement homogène. En trait continu ( $\longrightarrow \longrightarrow$ ) nous avons reproduit la courbe théorique relative au dioptre air-verre parfaitement plan. Les courbes de symboles respectifs $(-\infty)$ et $(-+-)$ sont afférentes à l'expression (3) et à un dioptre rugueux de rugosité quadratique moyenne $\sigma$ égale respectivement à $10 \AA$ et $40 \AA$. A titre indicatif, on notera que les rugosités font déjà chuter l'intensité réfléchie d'un facteur 10 lorsque l'angle d'incidence atteint $, 4,68 \theta_{\mathrm{c}}$ et $1,37 \theta_{\mathrm{c}}$ respectivement. On remarquera aussi la déformation très nette de la courbe $I \rightarrow f(\theta)$ pour le voisinage même de $\theta_{\mathrm{c}}$ lorsque $\sigma$ vaut $40 \AA$. Dans ce cas, pour la simulation de la courbe expérimentale, la contribution des rugosités peut devenir comparable, voire supérieure, à celle d'une couche superficielle de densité inférieure à la masse.

[Theoretical influence of surface roughness upon X-ray specular reflectance in the case of a perfectly homogeneous glass. The full line (with points) is drawn for a perfectly flat air-glass interface. Circles and crosses refer to interfaces with respectively 10 and $40 \AA$ r.m.s. roughness. So, the reflectance is falling by a factor of 10 , when the grazing angle is respectively $4.68 \theta_{\mathrm{c}}$ and $1.37 \theta_{\mathrm{c}}$. Observe also, in the last case, the departure of the $I \rightarrow f(\theta)$ curve near $\left.\theta_{\mathrm{c}} \cdot\right]$

des temps de calcul nettement plus courts. Par ailleurs on ne constate pas de divergences intempestives dans le calcul de la valeur de l'intensité réfléchie à $\theta=\theta_{\mathrm{c}}$ ou bien à $\theta$ très élevé, lorsque la rugosité devient assez forte. De telles divergences apparaissent avec le modèle de la couche de passage, pour $\sigma$ supérieur à $40 \AA$ environ, avec la méthode de calcul numérique de type Runge et Kutta utilisée (voir par exemple A. Angot Complément de mathématiques Revue d'Optique 5e édition, Paris (1965) page 788).

A titre de comparaison nous rappellerons ici les diverses expressions déjà trouvées (P. Croce, $L$. Névot [13]) pour l'intensité réfléchie spéculairement par un dioptre rugueux, lorsque le spectre de rugosité se caractérise par une prédominance des basses fréquences spatiales (4) ou au contraire par celle des hautes fréquences (5), soit respectivement :

$$
\begin{gathered}
I_{\mathrm{R}}=I_{\mathrm{I}}(\theta) \exp \left[-16 \pi^{2} K_{1 n}^{2}\left\langle Z_{\mathrm{D}}^{2}\right\rangle\right] \\
I_{\mathrm{R}}=I_{\mathrm{I}}(\theta)\left|1+\exp \left[-2 \pi^{2}\left\langle Z_{\mathrm{D}}^{2}\right\rangle\left(K_{1 n}+K_{2 n}\right)^{2}\right]-\exp \left[-2 \pi^{2}\left\langle Z_{\mathrm{D}}^{2}\right\rangle\left(K_{2 n}-K_{1 n}\right)^{2}\right]\right|^{2} .
\end{gathered}
$$

On peut remarquer que ces diverses expressions (3), (4), (5) sont sensiblement équivalentes lorsque $\left(K_{2 n}-K_{1 n}\right)$ devient suffisamment petit, c'est-à-dire dans la zone des incidences élevées, là où le phénomène de réfraction peut être négligé (soit pour $\theta \gtrsim 4 \theta_{\mathrm{c}}$, voir P. Croce, L. Névot [30]). Par contre dans la zone de réflexion totale $\left[\theta \lesssim \theta_{\mathrm{c}}\right]$ de fortes différences se manifestent, ainsi qu'on peut le constater sur les courbes de la figure 3 . Ces courbes sont afférentes aux expressions (3). (4) et (5) dans le cas d'une surface d'or de forte rugosité $(\sigma=17,5 \AA)$. On retrouve un assez bon accord entre l'expression (3) et le modèle de couche de passage fonction-erreur, ainsi que nous l'avons écrit précédemment. Par contre les expressions (4) et (5) conduisent à une atténuation nettement trop forte, ou au contraire trop faible, ce désaccord étant d'autant plus prononcé que la rugosité est élevée. Ainsi, dans le cas de l'expression (5), il devient 


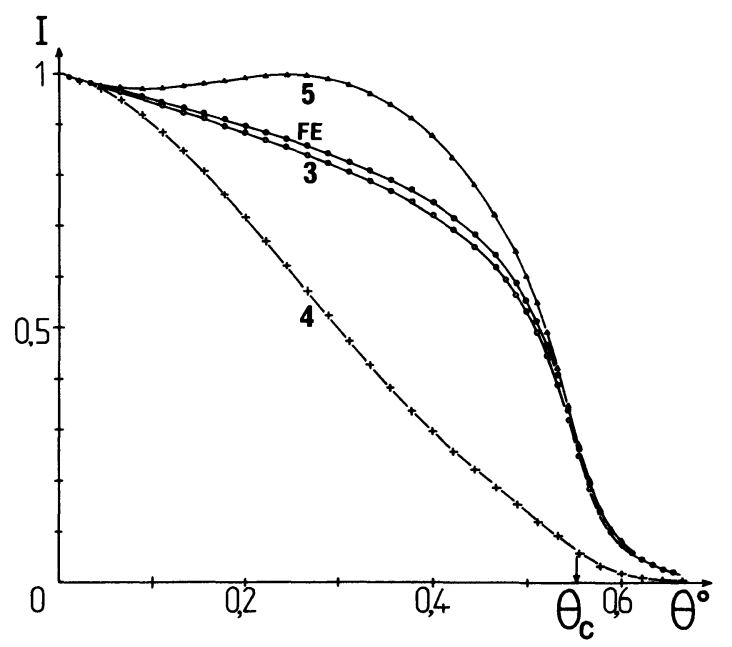

même possible d'obtenir des intensités relatives supérieures à 1 (?), cette anomalie intervenant d'autant plus facilement, à $\sigma$ donné, que l'absorption est faible.

2.2 CAS D'UN EMPILEMENT DE COUCHES DÉLIMITÉES PAR DES INTERFACES RUGUEUX. - Pour étendre le cas précédent à celui d'un empilement de couches il est nécessaire d'établir les expressions des amplitudes des champs électriques incident et réfléchi en remontant du substrat vers le vide. Ceci exige la connaissance de $t_{\mathrm{R}}$. Or la contribution à la correction de $t$, imputable aux ondes diffractées, est du même ordre que celle due aux ondes principales. Cette difficulté
Fig. 3. - Analyse comparative de la forme des courbes de réflexion spéculaire, dans la zone de réflexion totale, fournie par les expressions théoriques (3), (4) et (5). On considère ici un matériau (or) de forte densité électronique et forte absorption $\left(\delta=46,5 \times 10^{-6}\right.$, $\beta=4,9 \times 10^{-6}$ pour le rayonnement $\mathrm{CuK}_{\alpha_{1}}$ ), et de rugosité élevée $(\sigma=17,5 \AA)$. Pour la courbe notée F.E., le calcul de l'intensité est effectué en assimilant l'interface rugueux air-or à une couche de passage dans laquelle l'indice de réfraction varie entre 1 et la valeur massique de l'or, suivant la fonction-erreur.

[X.S.R. (X-ray specular reflection) curves in the total reflection range as derived from theoretical expressions (3), (4) and (5). In order to make the discrepancy most accentuated, we have considered a high r.m.s. roughness value $(17.5 \AA)$ and a material (gold) having a high electronic density, and a high absorption for the $\mathrm{CuK}_{\alpha_{1}}$ radiation. The F.E. curve is computed looking on the rough air-gold interface as a transition layer whose refractive index decreases from unity to the bulk value according to an errorfunction.]

peut être tournée en remarquant que l'effet des ondes diffractées s'annule dans l'expression de $1+R-T$ (voir les expressions (20) et $\left(20^{\prime}\right)$ de l'article de $\mathbf{P}$. Croce [26]). On en déduit par suite :

$$
1+R_{1 \mathrm{ss}}-T_{1 \mathrm{ss}}=-2 \pi^{2} h^{2} K_{1 n}\left(K_{1 n}-K_{2 n}\right) .
$$

Ce résultat peut se retrouver à partir des expressions générales (1). Si on considère le dioptre idéal dans le milieu 1, les expressions des champs $\mathbf{E}_{\mathrm{I}, 1}$ et $\mathbf{E}_{\mathbf{I}, 2}$ s'écrivent comme suit, les coefficients de réflexion et transmission $r_{1}, t_{1}$ étant afférents au passage du milieu 1 vers le milieu 2, et ceux notés $r_{2}, t_{2}$ au passage inverse (ce qui implique par conséquent $r_{2}=-r_{1}$ ):

soit encore :

$$
\begin{aligned}
& \mathbf{E}_{\mathrm{I}, 1}=t_{1} \mathrm{e}^{2 \pi i\left(K_{2 n} Z-K_{\mathrm{t}} X\right)} \mathbf{U}_{\mathrm{Y}} \cong t_{1}\left(1+2 \pi i K_{2 n} Z\right) \mathrm{e}^{-2 \pi i K_{\mathrm{t}} X} \mathbf{U}_{\mathrm{Y}} \\
& \mathbf{E}_{\mathrm{t}, 2}=\left[\mathrm{e}^{2 \pi i\left(-K_{2 n} Z-K_{\mathrm{t}} X\right)}+r_{2} \mathrm{e}^{2 \pi i\left(K_{2 n} Z-K_{\mathrm{t}} X\right)}\right] \mathbf{U}_{\mathrm{Y}}
\end{aligned}
$$

$$
\mathbf{E}_{\mathbf{1}, 2} \cong\left(t_{2}-2 \pi i K_{2 n} Z t_{1}\right) \mathrm{e}^{-2 \pi i K_{t} X} \mathbf{U}_{\mathbf{Y}}
$$

En remarquant que $t_{1} / K_{1 n}=t_{2} / K_{2 n}$, l'expression de $\left(1+r_{\mathrm{R}}-t_{\mathrm{R}}\right)$ se met sous la forme :

$$
1+r_{\mathrm{R}}-t_{\mathrm{R}}=i \pi \int\left[\mathbf{E}_{\mathrm{R}} \cdot 4 \pi i Z \mathbf{U}_{\mathrm{Y}}\right]\left(K_{1}^{2}-K_{2}^{2}\right) \mathrm{e}^{-2 \pi i K_{\mathrm{t}} X} \mathrm{~d} Z
$$

Les termes du deuxième ordre de cette expression proviennent soit des termes d'ordre 1 de $E_{\mathrm{R}}$ et d'ordre 0 de $E_{\mathrm{I}}$ (lequel est d'ailleurs nul dans la formule cidessus) soit de ceux d'ordre 0 de $E_{\mathrm{R}}$ et d'ordre 1 de $E_{\mathrm{I}}$ : d'où la disparition de l'effet des ondes diffractées dans $1+R_{1 \mathrm{ss}}-T_{1 \mathrm{ss}}$.

Quand $\left(K_{2}^{2}-K_{1}^{2}\right)$ tend vers $0,\left(1+r_{\mathrm{R}}-t_{\mathrm{R}}\right)$ tend donc vers 0 mais seulement au premier ordre.

Nous considérerons par suite que $t_{\mathrm{R}}$ est sensiblement égal à $\left(1+r_{R}\right)$. Lorsqu'on se trouve en présence de plusieurs couches, ou milieux $M_{\mathrm{j}}$, on peut décomposer le champ électromagnétique, au niveau de chaque interface, en un champ incident (désigné par $a$ ou $\alpha$, voir schéma 3 ) et un champ réfléchi ( $b$ ou $\beta$ ), en négligeant les champs diffractés. L'analyse s'effectue
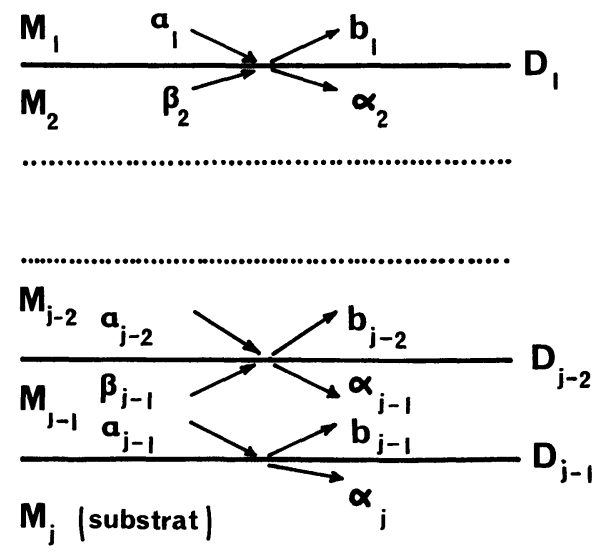

Schéma 3. 
alors en remontant l'empilement à partir de la dernière couche $M_{\mathrm{j}-1}$, le substrat étant noté $M_{\mathrm{j}}$. Considérons les ondes en présence au niveau de l'interface $D_{j-1}$. Du fait du substrat il n'y a qu'une onde transmise $\alpha_{\mathbf{j}}$. Si l'on se fixe à priori la valeur $a_{\mathbf{j}-1}$ de l'onde incidente, les formules établies précédemment en 2.1 permettent de connaître l'onde réfléchie $b_{\mathbf{j}-1}$.

On calcule ensuite les amplitudes $\alpha_{j-1}$ et $\beta_{j-1}$ de ces deux ondes pour la même couche $M_{\mathrm{j}-1}$ mais au niveau de l'autre interface $D_{j-2}$.

Le champ électromagnétique au voisinage de $D_{j-2}$ est considéré comme engendré par deux sources : les ondes $\beta_{\mathrm{j}-1}$ et $a_{\mathrm{j}-2}$, cette dernière étant déterminée à partir de $\alpha_{j-1}$. Soit $r_{R, j-2}$ le coefficient de réflexion pour une onde incidente $a_{\mathrm{j}-2}$ uniquement sur le dioptre $D_{j-2}$. Le coefficient de transmission s'écrira alors $1+r_{\mathrm{R}, \mathbf{j}-2}$. La contribution de $\beta_{\mathbf{j}-1}$ à $\alpha_{\mathbf{j}-1}$ est donnée par $-r_{\mathrm{R}, \mathrm{j}-2} \beta_{\mathrm{j}-1}$ et celle de $a_{\mathrm{j}-2}$ par $\left(1+r_{\mathrm{R}, \mathrm{j}-2}\right) a_{\mathrm{j}-2}$, soit par conséquent :

$$
\alpha_{\mathrm{j}-1}=\left(1+r_{\mathrm{R}, \mathrm{j}-2}\right) a_{\mathrm{j}-2}-r_{\mathrm{R}, \mathrm{j}-2} \beta_{\mathrm{j}-1}
$$

et, par suite :

$$
a_{\mathrm{j}-2}=\frac{\alpha_{\mathrm{j}-1}+r_{\mathrm{R}, \mathrm{j}-2} \beta_{\mathrm{j}-1}}{1+r_{\mathrm{R}, \mathrm{j}-2}}
$$

On en déduit ensuite $b_{\mathbf{j}-2}$ par la relation

$$
b_{\mathbf{j}-2}=\alpha_{\mathrm{j}-1}+\beta_{\mathrm{j}-1}-a_{\mathrm{j}-2} .
$$

On peut remonter ainsi de proche en proche jusqu'au premier interface $D_{1}$ et obtenir la valeur de la première onde incidente $a_{1}$. Notons qu'il est souvent nécessaire de prendre son amplitude égale à 1 . On divisera alors les valeurs de $a, b, \alpha$ et $\beta$ par $a_{1}$. Ceci fournit en particulier la valeur du coefficient de réflexion $b_{1} / a_{1}$ de l'empilement, mais aussi la valeur du champ dans chaque milieu ainsi que sa décomposition en ondes planes, renseignements extrêmement utiles pour l'étude suivante.

On remarquera que cette technique de programmation est très générale, et qu'elle peut s'adapter aussi bien aux empilements de couches minces avec interfaces uniquement de type rugueux (films réalisés, par exemple, dans une cloche à vide par déposition successive de composés différents, dans la mesure où il n'existe pas de diffusion de matière entre eux, ou bien encore films de type Langmuir-Blodgett) qu'à ceux délimités par des interfaces mixtes (c'est-à-dire à la fois rugueux et diffus) et stratifiés. En effet, pour ce dernier cas, il est possible de restituer la variation de l'indice de réfraction dans l'interface à l'aide d'un empilement de strates élémentaires, chaque strate ayant une épaisseur suffisamment faible et jouant le rôle d'un milieu $M_{\mathrm{j}}$ comme précédemment. Dans le chapitre suivant nous présentons une autre façon de traiter la réflexion spéculaire engendrée par une couche de passage stratifiée. Bien qu'approchée elle a cependant le mérite de conduire à une formula- tion explicite relativement simple de l'intensité réfléchie.

2.3 CAS D'UN INTERFACE DIFFUS, MAIS STRATIFIÉ - Nous examinons maintenant le cas où il existe, entre la masse de l'échantillon et sa surface, une couche de passage stratifiée, tous les points de même cote $Z$ étant caractérisés par la même valeur de constante diélectrique $n^{2}(Z)$. Ainsi qu'il a déjà été montré par P. Croce ([27], formule (3)), dès que l'on connaît ou que l'on a une estimation de la répartition du champ électrique $\mathbf{E}_{\mathrm{R}}(Z)$ qui règne dans cette zone de perturbation, il devient possible de déterminer son effet sur le coefficient de réflexion. En fait, cela suppose le problème résolu. On notera cependant que si l'épaisseur de la zone perturbée est faible devant, ce que nous appellerons, la longueur d'onde apparente, c'est-à-dire $1 / K_{2 n}$ si $\mathbf{K}_{2}$ représente le vecteur d'onde dans le milieu considéré, on peut admettre que $\mathbf{E}_{\mathrm{R}}(Z)$ est à peu près égal à $E_{\mathrm{I}}(\mathbf{Z})$ (champ qui règne dans le milieu homogène) même si les variations d'indice sont fortes. Ceci a déjà été discuté dans l'article cité précédemment. Nous en donnerons ici une explication plus physique.

Supposons tout d'abord la zone de perturbation d'épaisseur $\Delta Z$ suffisamment faible pour se comporter comme un plan de sources dipolaires de moment dipolaire :

$$
\frac{1}{4 \pi}\left[n_{\mathrm{R}}^{2}(Z)-n_{\mathrm{I}}^{2}(Z)\right] \mathbf{E}_{\mathrm{R}}(0,0, Z) \mathrm{e}^{2 \pi i \mathbf{K}_{\mathrm{t}} \cdot \mathbf{r}} \Delta Z \mathrm{~d} S .
$$

Montrons que dans ce cas $\mathbf{E}_{\mathbf{R}}(Z)$ est très voisin de $\mathbf{E}_{\mathrm{l}}(Z)$. Si la condition $\mathbf{E}_{\mathrm{l}} \cdot \mathbf{K}_{\mathrm{t}}=0$ est satisfaite, les sources dipolaires n'engendreront qu'un courant électrique et ne créeront aucune charge électrique moyenne : en effet, les dipôles qui sont alignés en projection sur le plan XOY sont de même phase et les charges des extrémités se détruisent. Les raisonnements classiques en électrostatique et en électrodynamique montrent que la composante principale du champ électrique n'est pas perturbée quand on traverse perpendiculairement cette couche, tandis que le champ magnétique subit une discontinuité d'amplitude, discontinuité qui ne produira d'effet sur le champ électrique que lorsqu'on s'éloigne de la couche d'une distance de l'ordre de la longueur d'onde apparente.

La condition $\mathbf{E}_{\mathbf{l}} \cdot \mathbf{K}_{\mathrm{t}}=0$ se trouve satisfaite rigoureusement si l'onde est du type « $\mathrm{s}$ » (champ électrique perpendiculaire au plan d'incidence). Elle l'est aussi approximativement pour le cas « $\mathrm{p}$ » (champ parallèle au plan d'incidence) lorsque l'onde est très rasante : la projection de $\mathbf{E}_{\mathbf{I}}$ dans le plan XOY est alors presque nulle. On remarquera que pour les rayons $X$ cette condition est d'autant mieux satisfaite du fait même de la réfraction, puisque, l'indice étant inférieur à 1 , l'onde en passant du vide dans la matière, tend à devenir plus rasante. Un tel résultat peut s'étendre évidemment au cas où l'épaisseur de la zone de per- 
turbation est plus épaisse, en restant toutefois petite devant la longueur d'onde apparente.

Remarquons que l'on vient seulement de montrer que la répartition $\mathbf{E}_{\mathrm{I}}(\mathbf{r})$ représente celle de $\mathbf{E}_{\mathrm{R}}(\mathbf{r})$ à un facteur près, du côté du dioptre opposé à celui d'où provient l'onde incidente puisque ces champs doivent correspondre à des ondes incidentes d'amplitude unité. Or nous venons de voir que le champ magnétique est fortement changé, ce qui implique aussi un fort changement du coefficient de transmission $t \cong 1+r$. On peut tenir compte de ce changement de façon automatique en écrivant $E_{\mathrm{R}}$ sous la forme

$$
E_{\mathrm{R}}=\frac{1+r_{\mathrm{R}}}{1+r_{\mathbf{I}}} E_{\mathbf{I}}
$$

puisque nous avons admis que le champ électrique était pratiquement le même de part et d'autre du dioptre, et que ce champ vaut $\left(1+r_{1}\right)$ dans le cas idéal et $\left(1+r_{\mathrm{R}}\right)$ dans le cas réel (les valeurs du coefficient de réflexion sont pratiquement les mêmes pour les deux types de polarisation $《 s \|$ et $« p$ i).

Dans l'article antérieur (P. Croce [27]), l'effet de la rugosité était traité de la même manière que celui d'une couche de passage. Ici, au contraire, on essaie de dissocier le rôle des rugosités de celui des inhomogénéités. En effet, dans la formulation précédente où $\mathcal{F}$ représente la correction par rapport au cas idéal [formules (2a) ou (4)], $\mathfrak{F}$ se rapproche fortement de 1 quand le coefficient de réflexion devient très faible (c'est-à-dire pour les incidences élevées, là où les rugosités ont une contribution prépondérante) et par conséquent doit être déterminé de façon très précise. Pour utiliser à nouveau l'expression ( $\left.2^{\prime}\right)$ que nous venons d'établir en 2.1 en tant que contribution des rugosités, il est nécessaire, d'une part, de prendre le plan de référence $Z=0$ du dioptre idéal de telle sorte que la surface rugueuse soit entièrement du côté $Z>0$, puis de faire tendre ce plan vers le plan moyen des rugosités, et d'autre part d'admettre que dans la zone des rugosités l'indice est sensiblement constant (nous l'appellerons $n_{0}$ par la suite).

On notera que pour l'intégration dans le milieu 2, à partir de l'expression générale (1) pour $Z>Z_{\mathrm{D}}$, le champ réel est défini par :

$$
\mathbf{E}_{\mathrm{R}, 2}=\left(1+r_{\mathrm{R}}\right) \mathrm{e}^{2 \pi i K_{2 n} Z} \mathrm{e}^{2 \pi i K_{\mathrm{t}} X} \mathbf{U}_{\mathbf{Y}}
$$

puisque le champ idéal s'exprime par :

$$
\mathbf{E}_{1,2}=\left(1+r_{\mathrm{I}}\right) \mathrm{e}^{2 \pi i K_{2 n} Z} \mathrm{e}^{-2 \pi i K_{\mathrm{t}} X} \mathbf{U}_{\mathbf{Y}} .
$$

Par suite il suffit de rajouter au deuxième membre de l'expression (2) le terme :

$$
i \pi \frac{K_{0}^{2}}{K_{1 n}}\left(1+r_{\mathrm{I}}\right)\left(1+r_{\mathrm{R}}\right)\left\langle\int_{Z_{\mathrm{D}}}^{\infty}\left(n^{2}(Z)-n_{2}^{2}\right) \mathrm{e}^{4 \pi i K_{2 n} Z} \mathrm{~d} Z\right\rangle .
$$

En effectuant l'intégration par parties on obtient pour ce terme :

$$
i \pi \frac{2 K_{0}^{2}}{K_{1 n}+K_{2 n}}\left[-\frac{\left(n_{0}^{2}-n_{2}^{2}\right)\left\langle\mathrm{e}^{+4 \pi i K_{2 n} Z_{\mathrm{D}}}\right\rangle}{4 \pi i K_{2 n}}-\left\langle\int_{Z_{\mathrm{D}}}^{\infty} \frac{\mathrm{e}^{4 \pi i K_{2 n} Z}}{4 \pi i K_{2 n}} \mathrm{~d}\left(n^{2}\right)\right\rangle\right]\left(1+r_{\mathrm{R}}\right) .
$$

Soit finalement (6) :

$$
\frac{K_{0}^{2}}{2 K_{2 n}\left(K_{1 n}+K_{2 n}\right)}\left[\left(n_{2}^{2}-n_{0}^{2}\right) \mathrm{e}^{-8 \pi^{2} K_{2 n}^{2}<Z_{D}>}-\int_{0}^{\infty} \mathrm{e}^{4 \pi i K_{2 n} Z} \mathrm{~d}\left(n^{2}\right)\right]\left(1+r_{\mathrm{R}}\right) .
$$

Pour poursuivre le calcul il est nécessaire de préciser la variation de la constante diélectrique $n^{2}(Z)$ dans la zone de perturbation, où du moins, cette variation n'étant pas connue, d'établir à l'aide de fonctions simples, un modèle qui puisse rendre compte des résultats expérimentaux, en introduisant un nombre de paramètres minimal.

Auparavant, nous devons nous interroger sur le domaine de validité de l'approximation adoptée pour $E_{\mathrm{R}}(\mathbf{r})$.

L'équation différentielle qui régit la propagation d'une onde de type « $s$ » dans un milieu stratifié de constante diélectrique $n_{\mathrm{R}}^{2}(Z)$ s'écrit :

$$
\frac{\partial^{2} E}{\partial Z^{2}}+4 \pi^{2}\left[\frac{n_{\mathrm{R}}^{2}(Z)}{\lambda^{2}}-K_{\mathrm{t}}^{2}\right] E=0
$$

Soit encore, en introduisant la variation de la constante diélectrique par rapport au milieu idéal

$$
\begin{gathered}
\Delta \varepsilon(Z)=n_{\mathrm{R}}^{2}(Z)-n_{\mathrm{I}}^{2}(Z) \\
\frac{\partial^{2} E}{\partial Z^{2}}+4 \pi^{2} K_{n_{1} \mathrm{I}}^{2} E=-4 \pi^{2} \frac{\Delta \varepsilon(Z)}{\lambda^{2}} E .
\end{gathered}
$$

Rappelons que $\Delta \varepsilon(Z)$ n'est différent de zéro que pour $Z \gtrsim 0$ sur une profondeur que nous supposerons finie et égale à $h$. 
La solution de l'équation (7) peut s'exprimer à partir de celle de l'équation sans second membre, soit $\exp \left(2 \pi i K_{n, \mathrm{I}} Z\right)$, sous la forme :

$$
\begin{aligned}
E_{\mathrm{R}}(Z) & =\mathrm{e}^{2 \pi i K_{n, \mathrm{I}} Z}- \\
& -\int_{+\infty}^{Z} \frac{\sin 2 \pi K_{n, \mathrm{I}}(Z-\zeta)}{2 \pi K_{n, \mathrm{I}}} \frac{4 \pi^{2} \Delta \varepsilon(\zeta)}{\lambda^{2}} E_{\mathrm{R}}(\zeta) \mathrm{d}(\zeta) .
\end{aligned}
$$

Montrons alors que si la condition (8) :

$$
\left|\frac{4 \pi^{2}}{\lambda^{2}} \int_{-\infty}^{\infty} Z \Delta \varepsilon(Z) \mathrm{d} Z\right| \ll 1
$$

est satisfaite nous pourrons remplacer $E_{\mathrm{R}}(Z)$ par sa valeur en $Z=0$ dans le cas idéal.

Si nous posons :

$$
S=\frac{\sin 2 \pi K_{n, \mathrm{I}}(Z-\zeta)}{2 \pi K_{n, \mathrm{I}}(Z-\zeta)},
$$

il nous suffit de montrer que | $S$ | est au plus de l'ordre de 1 .

Lorsque les angles d'attaque $\theta$ deviennent supérieurs à $\theta_{\mathrm{c}}, K_{n}$ étant réel, $|S|$ est alors toujours inférieur à 1 , et même très inférieur à 1 dès que l'on s'éloigne de $\theta_{\mathrm{c}}$. La condition restrictive imposée par (8) ne concerne par conséquent que la zone de réflexion totale et son voisinage immédiat.

Pour $\theta<\theta_{c}, K_{n}$ est imaginaire et cette fois $|S|$ est supérieur à 1 . Il restera cependant voisin de 1 si $\frac{2 \pi h}{\lambda} \sqrt{\cos ^{2} \theta-\cos ^{2} \theta_{c}}$ est petit devant 1. Cette dernière condition est par conséquent d'autant mieux vérifiée que l'on s'approche de la limite de réflexion totale. Dans le cas de la rasance pure $(\theta \cong 0)$, et ceci pour un verre de masse volumique $2,5 \mathrm{~g} / \mathrm{cm}^{3}$ (soit $\left.\theta_{\mathrm{c}} \cong 4 \mathrm{mrad}\right)$ attaqué par le rayonnement $\mathrm{CuK}_{\alpha_{1}}$ $(\lambda=1,5405 \AA)$, la condition s'exprime par $h<60 \AA$.

D'après ce que nous avons vu dans le chapitre 1 , les épaisseurs moyennes des couches superficielles engendrées par le polissage des surfaces de verre, sont de l'ordre de quelques dizaines, voire quelques centaines d'angströms. Ainsi, les valeurs indiquées par G. Kühnen [23] pour le verre BK7 $\left(e_{\mathrm{c}}=65 \AA\right.$ et $\left.\delta_{\mathrm{c}}=7,5 \times 10^{-6}\right)$ conduisent pour le premier membre de (8) à la valeur 0,077 . De même, celle que nous trouvons pour le verre B-1664 à l'aide du modèle à deux dioptres (voir Fig. 1) s'établit à 0,148 , l'épaisseur moyenne étant alors de $90 \AA$.

Nous pouvons donc considérer que nos approximations ne seront, en général, pleinement justifiées qu'à partir des incidences assez proches de la limite de réflexion totale.

$\mathrm{Eu}$ égard aux incidences les plus rasantes nous devons rappeler que les enregistrements expérimentaux $I \rightarrow f(\theta)$ ne doivent être pris en considération que pour $\theta>\theta_{\mathrm{g}}$, si $\theta_{\mathrm{g}}$ représente l'angle d'attaque minimum à partir duquel le faisceau incident est entièrement intercepté par l'échantillon. Ainsi, pour une ouverture de fente de $80 \mu \mathrm{m}$ et un échantillon de dimension latérale maximale de $5 \mathrm{~cm}, \theta_{\mathbf{g}}$ est égal à $1,6 \mathrm{mrad}$ soit $0,4 \theta_{\mathrm{c}}^{\text {verre }}$.

Comme nous l'avons écrit précédemment l'évaluation de l'expression (6) ne peut être effectuée que si l'on connaît la variation de $n^{2}(Z)$ entre la valeur $n_{0}^{2}$ qui caractérise la surface et celle, $n_{2}^{2}$, du matériau massif homogène. Nous avons déjà vu que l'interface rugueux peut être assimilé à une couche de passage où l'indice varie suivant la fonction-erreur entre les valeurs afférentes au vide d'une part et à la surface du matériau d'autre part. Si l'on envisage la formation au cours du polissage d'une couche superficielle suffisamment uniforme quant à son indice et à son épaisseur, la variation $n^{2}(Z)$ pour le passage couche-substrat pourra se représenter par une simple fonction-erreur, soit :

$$
F\left(Z-\tau_{2}, \sigma_{2}\right)=\frac{1}{\sigma_{2} \sqrt{2 \pi}} \int_{-\infty}^{Z-\tau_{2}} \exp \left(-\frac{u^{2}}{2 \sigma_{2}^{2}}\right) \mathrm{d} u
$$

où $\tau_{2}$ représente l'épaisseur moyenne de la couche superficielle, et $\sigma_{2}$ l'écart-type de la distribution gaussienne associée (Schéma 4).

$$
\begin{aligned}
& n^{2}(Z) \text { s'exprimera alors par : } \\
& \quad n^{2}(Z)=n_{0}^{2}+\left(n_{2}^{2}-n_{0}^{2}\right) F\left(Z-\tau_{2}, \sigma_{2}\right) .
\end{aligned}
$$

Dans le cas contraire, où la composition de la couche et (ou) sa compacité varient continûment à partir de la surface, conférant par là même un caractère nettement diffus à l'interface air-substrat, une simple loi exponentielle paraît plus indiquée, soit par suite (Schéma 5) :

$n^{2}(Z)=n_{0}^{2}+\left(n_{2}^{2}-n_{0}^{2}\right)\left(1-\mathrm{e}^{-Z / \tau_{1}}\right)$ avec $Z \geqslant 0$.

D'une façon générale, nous utiliserons donc comme expression de base de $n^{2}(Z)$ pour la zone de contamination (9) :

$$
\begin{aligned}
n^{2}(Z)=n_{0}^{2}+\left(n_{1}^{2}-\right. & \left.n_{0}^{2}\right)\left(1-\mathrm{e}^{-Z / \tau_{1}}\right)+ \\
& +\left(n_{2}^{2}-n_{1}^{2}\right) F\left(Z-\tau_{2}, \sigma_{2}\right) .
\end{aligned}
$$
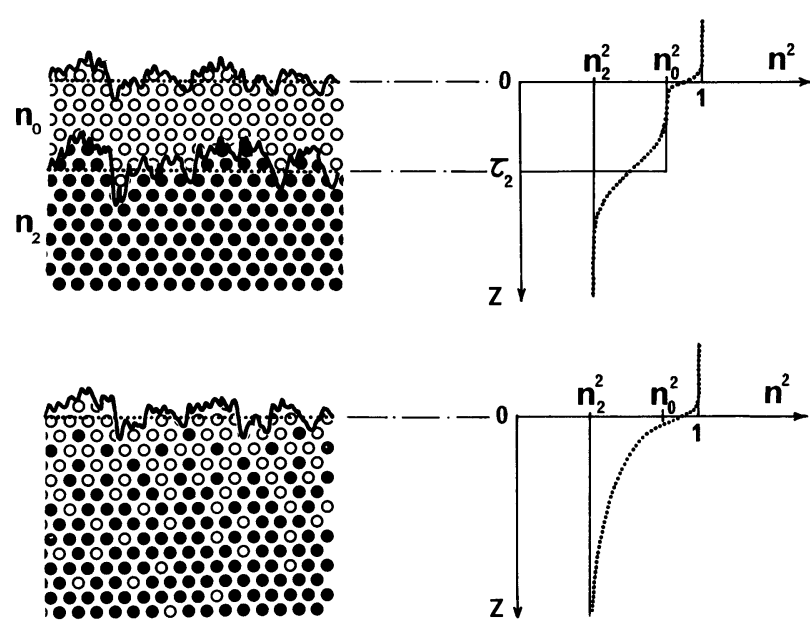

Schémas 4 et 5 . - Les profils $n^{2}(Z)$, représentés en pointillés, tiennent compte des rugosités de surface.

[Profiles $n^{2}(Z)$ shown in dotted lines allow for surface roughness.] 
Notons au passage que si l'on tient compte des rugosités de la surface, l'expression (9) se transforme comme suit (10) :

$$
n^{2}(Z)=1+\left(n_{0}^{2}-1\right) F\left(Z, \sigma_{1}\right)+\left(n_{1}^{2}-n_{0}^{2}\right)\left(1-\mathrm{e}^{-Z / \tau_{1}}\right)+\left(n_{2}^{2}-n_{1}^{2}\right) F\left(Z-\tau_{2}, \sigma_{2}\right) .
$$

Le résultat du calcul de l'expression (6) à partir de (9) peut se mettre sous la forme $\mathcal{A}\left(1+r_{R}\right)$ si l'on pose :

$$
\mathcal{A}=\frac{K_{0}^{2}}{2 K_{2 n}\left(K_{1 n}+K_{2 n}\right)}\left[\left(n_{2}^{2}-n_{0}^{2}\right) \mathrm{e}^{-8 \pi^{2} K_{2 n}^{2} \sigma_{1}^{2}}-\frac{\left(n_{1}^{2}-n_{0}^{2}\right)}{4 \pi i K_{2 n} \tau_{1}-1}-\left(n_{2}^{2}-n_{1}^{2}\right) \mathrm{e}^{-8 \pi^{2} \sigma_{2}^{2} K_{2 n}^{2}+4 \pi i K_{2 n} \tau_{2}}\right] .
$$

Compte tenu de (2), on obtient alors la relation :

$$
r_{\mathrm{R}} \mathrm{e}^{-2 \pi^{2}\left(\boldsymbol{K}_{1 n}-K_{2 n}\right)^{2} \sigma_{1}^{2}}=r_{\mathrm{I}} \mathrm{e}^{-2 \pi^{2}\left(\boldsymbol{K}_{1 n}+K_{2 n}\right)^{2} \sigma_{1}^{2}}+\mathcal{A}\left(1+r_{\mathrm{R}}\right) .
$$

Soit finalement (11) :

$$
r_{\mathrm{R}}=r_{\mathrm{I}} \mathrm{e}^{-8 \pi^{2} \sigma_{1}^{2} K_{1 n} K_{2 n}}+\left(1+r_{\mathrm{R}}\right) \mathcal{A} \mathrm{e}^{2 \pi^{2}\left(K_{1 n}-K_{2 n}\right)^{2} \sigma_{\mathrm{I}}^{2}} .
$$

L'influence, sur la forme de la courbe de réflexion spéculaire, des divers paramètres introduits dans l'expression (10) a déjà été discutée par P. Croce [27].

Rappelons que pour la zone des incidences élevées l'effet prépondérant est celui de la rugosité de surface $\sigma_{1}$. Par contre pour le voisinage immédiat de la limite de réflexion totale $\left(\theta \gtrsim \theta_{\mathrm{c}}\right)$ interviennent principalement $: n_{0}$, indice de la surface, et la variation totale de constante diélectrique intégrée, soit :

$$
\chi=\int_{-\infty}^{+\infty} \Delta \varepsilon(Z) \mathrm{d} Z=\tau_{1}\left(n_{0}^{2}-n_{1}^{2}\right)+\tau_{2}\left(n_{1}^{2}-n_{2}^{2}\right) .
$$

Par la suite nous utiliserons la variation totale de densité intégrée, laquelle est directement proportionnelle à :

$$
J=\int_{-\infty}^{+\infty} \Delta \delta(Z) \mathrm{d} Z=-\tau_{1}\left(\delta_{1}-\delta_{0}\right)-\tau_{2}\left(\delta_{2}-\delta_{1}\right) .
$$

Une valeur négative de $J$ traduira donc, ici, un défaut global de compacité (et par suite la prépondérance d'une certaine microporosité ou lacunisation de la couche de passage). Au contraire, une valeur positive impliquera l'existence d'une zone, assez épaisse, plus dense que la masse.

Dans la zone des incidences moyennes les paramètres $\tau_{2}$ et $\sigma_{2}$ qui caractérisent la couche superficielle limite deviennent essentiels. En effet, $\tau_{2}$ fixe la position des franges d'interférence (ou oscillations d'intensité) et $\sigma_{2}$ détermine leur contraste.

Par conséquent, l'analyse de la courbe complète de réflexion spéculaire doit permettre l'évaluation des divers paramètres de (10) de façon univoque.

Précisons que, si dans le cas des verres polis il n'est pas nécessaire d'introduire d'autres paramètres pour rendre compte parfaitement des courbes expérimentales, il est toutefois possible d'injecter dans (10) autant de fonctions-erreur, et par suite d'ensembles de paramètres $\left(n_{\mathrm{i}}, \sigma_{\mathrm{i}}, \tau_{\mathrm{i}}\right)$ qu'il se présente d'interfaces dans la zone de perturbation. Ainsi, dans le cas des surfaces d'acier ou de fer passivées, on invoque fréquemment la formation d'une double couche, l'une d'hydroxyde et l'autre d'oxyde; il est alors nécessaire d'introduire les trois interfaces airhydroxyde, hydroxyde-oxyde et oxyde-masse.

Rappelons aussi que la validité de notre théorie, pour ce qui concerne la zone de réflexion totale et son voisinage immédiat, repose sur l'inégalité (8). Compte tenu de (10) celle-ci s'exprime par :

$$
\begin{aligned}
\frac{4 \pi^{2}}{\lambda^{2}} \mid\left(n_{0}^{2}-n_{1}^{2}\right) \tau_{1}^{2}+ & \left(1-n_{0}^{2}\right) \frac{\sigma_{1}^{2}}{2}+ \\
& +\left(n_{1}^{2}-n_{2}^{2}\right)\left(\frac{\tau_{2}^{2}+\sigma_{2}^{2}}{2}\right) \mid \ll 1 .
\end{aligned}
$$

Pour la plupart des matériaux analysés au chapitre suivant cette condition sera amplement satisfaite, la valeur du premier membre de l'inégalité ne dépassant pas quelques \%. Par contre pour les matériaux fortement densifiés, tels que la silice pure et le verre aluminosilicaté, cette valeur atteint 0,45 , ce qui limitera alors la zone de validité à $\theta>\theta_{\mathrm{c}}$.

3. Résultats expérimentaux. - Nous avons examiné différents verres silicatés (silice pure, borosilicate et alumino-silicate) après polissage sur poix (poix de Bourgogne jaune ou verte, poix de Norvège noire) les poudres de polissage étant constituées d'oxydes de cérium, de fer ou d'étain et utilisées uniquement en milieu aqueux.

La planéité requise pour l'analyse était d'une frange au moins dans la plus grande dimension (soit $50 \mathrm{~mm}$ ).

Préalablement à l'étude par rayons $X$ rasants les surfaces ont été dégraissées à l'alcool pur, puis nettoyées à l'aide d'un coton imbibé d'une solution très diluée de R.B.S. et de carbonate de calcium, rincées ensuite sous eau déminéralisée et finalement séchées par centrifugation. Une autre méthode, toute aussi efficace pour ce type de verre, consiste à recouvrir la surface, une fois dégraissée, d'une couche de vernis 
que l'on arrache ensuite après durcissement (ce vernis est aussi utilisé pour les répliques en microscopie électronique).

L'ensemble instrumental a déjà été décrit par ailleurs (G. Devant [31], L. Névot [32]). Rappelons que les rotations de la platine porte-objet et du détecteur (compteur proportionnel) du goniomètre sont assurées par l'intermédiaire de moteurs pas à pas avec une précision angulaire de 1 ", ces moteurs étant asservis à un ordinateur. Les flux enregistrés varient, grâce

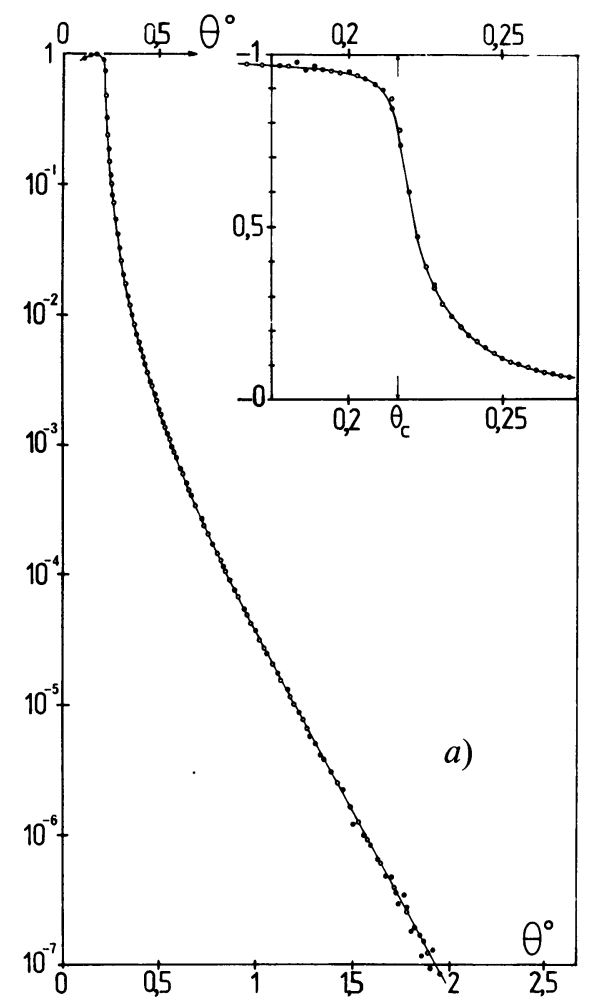

Fig. 4. - $-a)$ Analyse de la courbe expérimentale de réflexion spéculaire $I \rightarrow f(\theta)$ fournie par un verre de silice (homosil), poli à l'oxyde de cérium sur poix de Norvège noire. Les valeurs théoriques représentées par des points à centre clair $(O \circ O)$ sont issues de notre modèle d'interface, diffus (rugueux et inhomogène) et stratifié, défini au chapitre 2.3. [expression (11)]. b) Profils $\delta(Z)$ issus de la simulation de la courbe expérimentale précédente et correspondant aux expressions $\left(10^{*}\right)$ (trait continu) et $\left(9^{*}\right)$ (tirets). Rappelons que $\delta$ est directement proportionnel à la densité électronique $\eta$ (soit $\left(\rho Z^{*} / A\right)$ ). Dans le cas du rayonnement $\mathrm{CuK}_{a_{1}}$, la relation s'écrit : $\delta=6,412 \times 10^{-6} \eta$ si $\eta$ est exprimé en électrons par $\mathrm{cm}^{3}$. On constate ainsi la formation pendant le polissage de deux zones de perturbation. La première, au voisinage immédiat de la surface (épaisseur moyenne d'une dizaine d'angströms), se caractérise par une densité plus faible que celle de la masse. La seconde, sous-jacente, est au contraire plus dense que la masse et s'étend sur une profondeur de plusieurs centaines d'angströms.

[a) XSR experimental curve from a silica glass (homosil) polished with cerium oxide on swedish pitch. Values shown as circles are computed from equation (11). b) Electronic density profile (E.D.P.) versus depth (as measured from the mean level of the rough surface) according to expression $\left(10^{*}\right)$ (full line) or $\left(9^{*}\right)$ (dashed line) with parameter values used in figure $4 a$. During glass polishing two perturbed zones arise : the outer layer about $10 \AA$ thick presents a density lower than the bulk, the latter occuring below is more dense than the bulk and extends down to hundreds of angströms deep.] à un jeu d'écrans calibrés, dans un rapport de 1 à $10^{-6}$ (voire $10^{-7}$ ) du flux mesuré dans le faisceau direct, la plage angulaire totale explorée ici ne dépassant pas 3 degrés environ et étant couverte par pas angulaire $\Delta \theta$ de $10^{\prime \prime}$ au voisinage de $\theta_{\mathrm{c}}$ et de $40^{\prime \prime}$ pour les incidences élevées.

3.1 VerRe De Silice PURE. - Sur la figure $4 a$ est présentée la courbe de réflexion spéculaire d'un verre de silice (homosil de chez Heraeus) poli sur poix de Norvège noire à l'oxyde de cérium (cerox 1670Rhone Poulenc) en milieux aqueux. Un accord quasi parfait est obtenu sur toute la plage angulaire analysée.

On notera que l'expression (10) de $n^{2}(Z)$ conduit aisément à celles de $\delta(Z)$ et de $\beta(Z)$, sachant que d'une façon générale on a : $\beta^{2} \ll \delta^{2} \ll 1$. On obtient par suite :

$$
\begin{aligned}
\delta(Z)=\delta_{0} F\left(Z, \sigma_{1}\right) & +\left(\delta_{1}-\delta_{0}\right)\left(1-\mathrm{e}^{-Z / \tau_{1}}\right)+ \\
& +\left(\delta_{2}-\delta_{1}\right) F\left(Z-\tau_{2}, \sigma_{2}\right)
\end{aligned}
$$

et l'expression homologue de $\beta(Z)$ en substituant $\beta_{\mathrm{i}}$ à $\delta_{\mathrm{i}}(i=0,1,2)$.

De même l'expression (9) de $n^{2}(Z)$, obtenue en supposant la surface libre parfaitement plane, conduit à :

$$
\begin{aligned}
\delta(Z)=\delta_{0}+\left(\delta_{1}-\right. & \left.\delta_{0}\right)\left(1-\mathrm{e}^{-Z / \tau_{1}}\right)+ \\
& +\left(\delta_{2}-\delta_{1}\right) F\left(Z-\tau_{2}, \sigma_{2}\right) .
\end{aligned}
$$

En gommant l'effet de moyenne introduit par les rugosités de surface, c'est-à-dire par la fonctionerreur $F\left(Z, \sigma_{1}\right)$, le profil issu de l'expression $\left(9^{*}\right)$ fait davantage ressortir les modifications éventuelles d'indice, donc de densité électronique, au voisinage de la surface.

Sur la figure $4 b$ les deux profils $\delta(Z)$ relatifs aux expressions respectives $\left(10^{*}\right)$ et $\left(9^{*}\right)$ sont représentés en trait continu et en tirets. Précisons d'autre part que nous utilisons la même loi de variation pour $\beta(Z)$ que celle de $\delta(Z)$, l'absorption n'ayant, pour les verres considérés, que peu d'effet sur la forme des courbes.

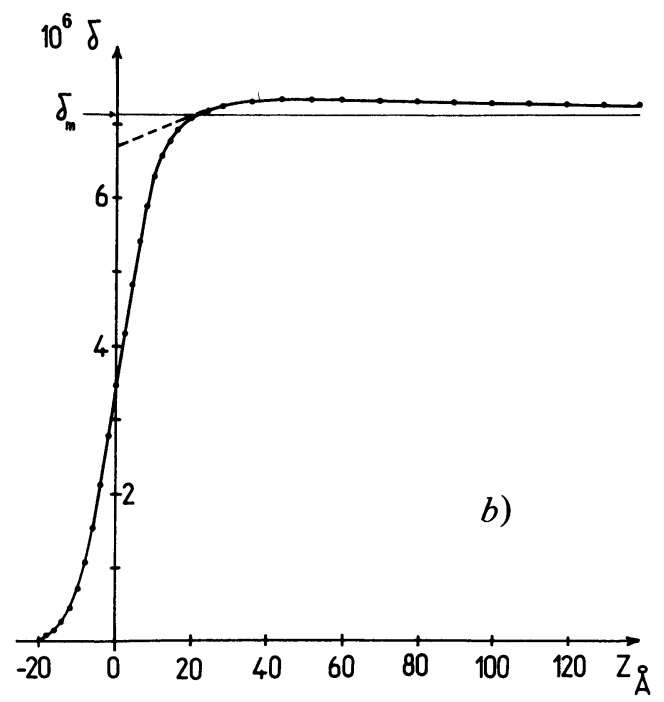


La surface de l'échantillon présentée ici se caractérise d'une part par une rugosité de $7,75 \AA$ et d'autre part par un indice $\delta$ de valeur inférieure à celle de la masse : soit $6,5 \times 10^{-6}$ au lieu de $7,12 \times 10^{-6}$. Ceci traduit l'existence d'une couche superficielle de densité, ou de compacité, inférieure à celle de la silice massique, couche vraisemblablement constituée de silice hydrolysée, et d'épaisseur très faible $\left(\tau_{2}=11 \AA\right)$. Immédiatement en dessous apparaît la densification de la silice, laquelle s'étend sur une profondeur de plusieurs centaines d'angströms. Le maximum de $\delta$ se situe à $51 \AA$ sous la surface moyenne et vaut $7,34 \times 10^{-6}$ (soit $\rho_{\max }=2,27 \mathrm{~g} / \mathrm{cm}^{3}$ ). $\delta(Z)$ diminue ensuite très lentement selon une loi exponentielle de constante $\tau_{1}$ égale à $225 \AA$.

Notons que les résultats précédents sont très voisins de ceux obtenus sur la silice polie à l'oxyde de fer sur poix de Bourgogne jaune (L. Névot [32]) : la rugosité était toutefois légèrement plus faible $(6,45 \AA)$ et la densification à la fois plus forte

$$
\left[\delta_{\max }=7,48 \times 10^{-6} \text { ou } \rho_{\max }=2,31 \mathrm{~g} / \mathrm{cm}^{3}\right]
$$

et moins profonde [maximum à $40 \AA$ sous la surface et $\left.\tau_{1}=160 \AA\right]$.

La densification de la silice vitreuse sous l'action de très fortes pressions exercées par l'intermédiaire de presses hydrauliques a été l'objet de nombreuses études depuis les travaux de Bridgman et Simon [33]. Ces derniers indiquent notamment que la densification permanente se manifeste à température ambiante dès que la pression appliquée dépasse $10^{10} \mathrm{Nm}^{-2}$ environ, la variation relative de densité étant ensuite d'autant plus élevée que la pression exercée est forte : elle serait de 6 à $7 \%$ pour une pression de $2 \times 10^{10} \mathrm{Nm}^{-2}$. Ils constatent en outre que la densification est d'autant plus faible que la teneur en ions alcalins augmente dans le verre de silice, ou que l'on passe de $\mathrm{Li}$ à $\mathrm{Na}$ et $\mathrm{K}$ respectivement (autrement dit plus le rayon de l'ion considéré est grand, moins la densification obtenue est forte, tout autre paramètre gardant la même valeur).

Mackenzie [34], outre l'effet de la pression (intensité et durée d'application) et celui de la température, souligne le rôle important des contraintes de cisaillement, lesquelles accroissent d'autant la densification finale obtenue à pression, ou à température, constante et abaissent notablement le seuil critique de densification permanente.

Or on conçoit aisément que le frottement des grains d'oxyde sur la surface de verre induise à la surface même une contrainte de cisaillement élevée. D'un autre côté la pression exercée localement par les grains, ceci sur une surface très petite car leurs dimensions moyennes sont inférieures au micron, peut fort bien représenter quelque $10^{9} \mathrm{Nm}^{-2}$ même lorsque la pression moyenne (ou pression nominale de polissage) transmise par l'intermédiaire du polissoir ne dépasse pas quelque $10^{3} \mathrm{Nm}^{-2}$ (H. Sakata [17]). La densification apparaît donc bien liée aux fortes pressions exercées localement par les grains d'oxyde. Les mesures ellipsométriques de Sakata confirment nettement, pour la silice pure, l'accroissement de cette densification moyenne lorsque la pression nominale de polissage augmente.

Ces mesures font apparaître en outre que pour les polissages effectués sous pression nominale supérieure à $6 \times 10^{3} \mathrm{Nm}^{-2}$, le produit $e \Delta n$, de l'épaisseur moyenne de la zone de densification à la variation relative d'indice $\Delta n$ par rapport à la masse, reste pratiquement constant.

Pour tenter la comparaison avec la variation d'indice (ou de densité) intégrée que l'on peut déduire des graphes $\delta(Z)$, il est nécessaire de connaître la loi de variation de l'indice de réfraction (dans le visible) avec la densité. Selon J. Arndt et Stöffler [35] cette loi peut être considérée comme linéaire pour les densités inférieures à 2,26 (ce qui correspond sensiblement au domaine exploré par Sakata) mais par contre au-delà, certaines irrégularités ou anomalies se manifestent, en particulier au voisinage des valeurs de densité afférentes aux phases cristallisées de la silice. On en déduit par suite la valeur $e \Delta \rho=17,5 \times 10^{-8} \mathrm{~g} / \mathrm{cm}^{2}$ pour Sakata, alors que nos deux exemples précédents conduisent à $16,4 \times 10^{-8}$ et $19,6 \times 10^{-8} \mathrm{~g} / \mathrm{cm}^{2}$ respectivement.

L'accord sur ce point est donc remarquable mais l'allure du graphe de $\delta(Z)$, qui se caractérise par une décroissance exponentielle très lente, montre bien qu'il est difficile d'attribuer une épaisseur et une densité moyennes à la zone de densification.

Pour éviter la densification superficielle de la silice vitreuse il apparaît nécessaire de diminuer la pression nominale de polissage et (ou) d'augmenter la viscosité $\mathrm{du}$ polissoir. Après avoir poli sur poix noire de Norvège à l'oxyde de cérium un échantillon cylindrique (diamètre de $50 \mathrm{~mm}$ ) de silice vitreuse, nous lui avons fait subir huit heures supplémentaires de polissage sur polissoir revêtu d'une épaisseur de $0,5 \mathrm{~mm}$ de téflon graphité à $30 \%$, la pression nominale étant d'autre part réduite à celle due au propre poids de l'échantillon (soit $65 \mathrm{Nm}^{-2}$ ). Pour cela, l'échantillon est introduit dans l'évidement cylindrique d'une pièce de silice en forme d'anneau de garde, de diamètre intérieur tout juste supérieur à $50 \mathrm{~mm}$. L'échantillon à polir se trouve entraîné par l'anneau dans son mouvement de va-et-vient sur le polissoir, tout en étant libre d'évoluer verticalement. Un tel système permet en outre d'éviter l'accumulation de produit de polissage sur les bords de la pièce à polir et par suite de diminuer son rabat (G. Otte [36-37]).

Le profil $\delta(Z)$ relatif à ce type de polissage est reproduit sur la figure 5 . On y remarque l'absence de densification. L'indice en surface reste sensiblement le même qu'auparavant $\left(6,3 \times 10^{-6}\right)$ mais l'épaisseur de la couche de silice hydrolysée est légèrement plus forte $\left(\tau_{2}=20 \AA\right)$. Notons en outre la diminution sensible de la rugosité $(6,3 \AA)$.

La densification superficielle de la silice polie peut 


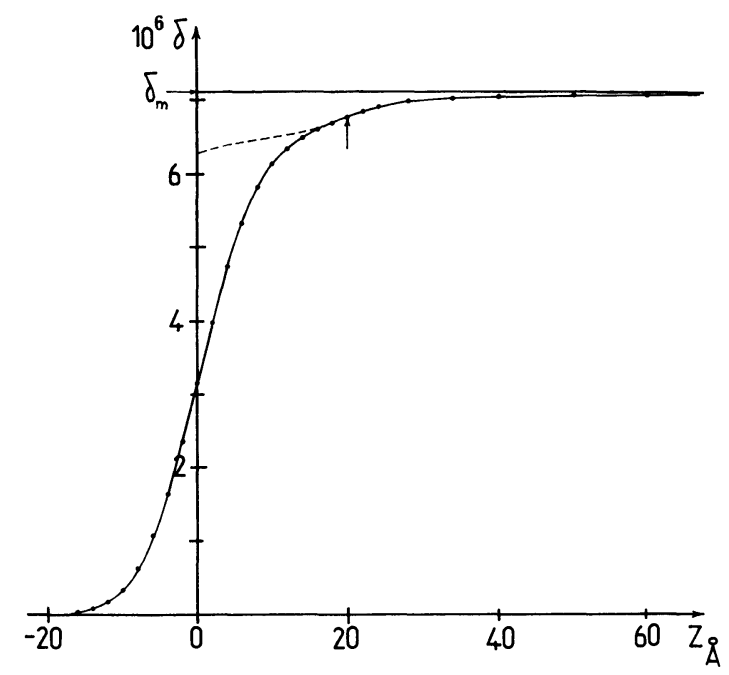

Fig. 5. - Profil $\delta(Z)$ d'un verre de silice ayant subi un polissage final sur téflon graphité pendant huit heures. La courbe en trait continu tient compte de l'effet de rugosité [expression $\left(10^{*}\right)$ ], celle en tirets [expression $\left(9^{*}\right)$ ] fait mieux ressortir la présence d'une couche superficielle d'épaisseur moyenne égale à $20 \AA$ (le point d'inflexion caractéristique de la deuxième fonction-erreur est repéré par la flèche verticale). On notera que la durée de ce polissage a été suffisante pour éliminer complètement la zone densifiée lors de l'ébauche préalable sur poix de Norvège noire.

[E.D.P. of a silica glass when the polishing is completed the last eight hours on graphite teflon. The full line takes into account the surface roughness (eq. $\left(10^{*}\right)$ ). The dashed one makes evident the outer layer about $20 \AA$ thick (the arrow points out the inflexion of the second error-function). Notice that the last polishing process has long enough to completely eliminate the compacted zone which appears on polishing with swedish pitch.]

être éliminée aussi par traitement thermique ainsi qu'on peut le constater sur les graphes $\delta(Z)$ de la figure 6 relatifs à l'échantillon, précédemment analysé en $4 b$, et soumis pendant une heure, à $300^{\circ} \mathrm{C}$ d'abord, puis à $400^{\circ} \mathrm{C}$. Le premier traitement laisse encore une certaine densification $\left(\delta_{\max }=7,274 \times 10^{-6}\right.$ soit $\rho_{\max }=2,25 \mathrm{~g} / \mathrm{cm}^{3}$ à $52 \AA$ sous la surface) mais sur une profondeur plus faible $\left(\tau_{1}=100 \AA\right)$. Par contre le second est suffisant pour faire disparaître cette densification résiduelle. Ce résultat est très différent de celui obtenu en ellipsométrie par Yokota et al. [16], lesquels constatent d'une part que la densification subsiste encore même si l'échantillon est porté à $500^{\circ} \mathrm{C}$ pendant une heure, et d'autre part que l'épaisseur moyenne de la couche densifiée croît fortement !

Nos résultats indiquent en outre que la pellicule de silice hydrolysée n'est pas affectée par ces traitements thermiques successifs, l'indice de surface et l'épaisseur $\tau_{2}$ restant inchangés. Par contre la rugosité est abaissée de façon appréciable de $7,75 \AA$ à $6,70 \AA$ puis $6,0 \AA$ respectivement.

Le fait de pouvoir, d'une part, densifier la silice vitreuse et, d'autre part, annuler cet effet par traitement thermique à température relativement basse (ce qui correspond à un processus de faible énergie d'activation) apparaît comme la conséquence de la grande flexibilité de la liaison $\mathrm{Si}-\mathrm{O}-\mathrm{Si}$. Ainsi, dans la silice non densifiée, l'angle de cette liaison varie de $120^{\circ}$ à $180^{\circ}$, la valeur optimum se situant à $144^{\circ}$ (Mozzi et Warren [38]). Selon Couty [39] la densification s'accompagne essentiellement d'une diminution de la valeur optimale, laquelle n'est plus égale qu'à $135^{\circ}$ pour la silice de masse volumique $2,555 \mathrm{~g} / \mathrm{cm}^{3}$. Par contre le tétraèdre $\mathrm{SiO}_{4}$ serait invariant.

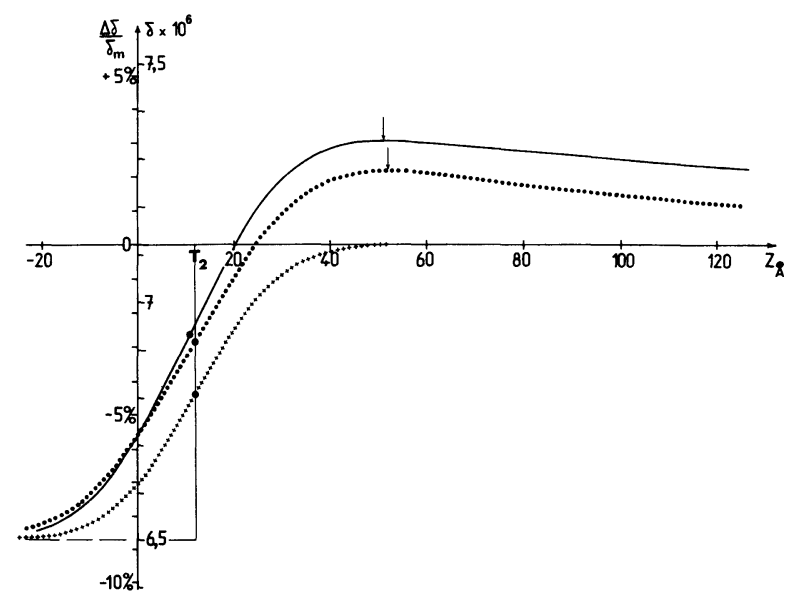

Fig. 6. - Influence de la température sur la densification d'un verre de silice poli à l'oxyde de cérium sur poix de Norvège noire. Les profils $\delta(Z)$ sont issus de l'expression $\left(9^{*}\right), \Delta \delta / \delta_{\mathrm{m}}$ indiquant la variation relative correspondante par rapport à la valeur massique. La courbe en trait plein est relative à l'échantillon brut de polissage. Celles de symboles respectifs $(\bullet \bullet \bullet \bullet)$ et $(++++)$ concernent le même échantillon porté pendant une heure à une température de $300^{\circ} \mathrm{C}$ puis $400^{\circ} \mathrm{C}$. Les flèches verticales repèrent ici les positions exactes du maximum de densification, la cote $Z$ des points renforcés donnant l'épaisseur moyenne $\tau_{2}$ de la couche limite superficielle.

[Temperature dependence of the compaction of silica glass polished with cerium oxide on a swedish pitch. E.D.P. $\delta(Z)$ refers to expression $\left(9^{*}\right)$. On the left side of the scale appears the relative density, with respect to the bulk value. The full line refers to the specimen as just polished. The other lines (points and crosses) relate to the same specimen when heated one hour at respectively 300 and $400^{\circ} \mathrm{C}$. Observe that, if the compaction of the sublayer progressively disappears, the mean thickness $\tau_{2}$ and the density of the outer layer don't change during heat treatment.

3.2 Alumino-silicate. - Ce type de verre se caractérise par une densité de 2,63 et une composition pondérale approximative de $60 \% \mathrm{SiO}_{2} 20 \% \mathrm{Al}_{2} \mathrm{O}_{3}$ et $20 \% \mathrm{CaO}$. Le polissage a été effectué manuellement sur poix de Bourgogne jaune, avec une suspension aqueuse d'oxyde de fer.

L'analyse pâr réflexion rasante de rayons $\mathrm{X}$ témoigne d'un comportement assez voisin de celui de la silice pure. Le graphe $\delta(Z)$ (courbe en trait plein des figures $7 a$ et $7 b$ ) résulte de l'existence d'une couche superficielle limite très mince $\left(\tau_{2}=21 \AA\right)$ de densité inférieure à celle de la masse $\left[\delta_{0}=6,6 \times 10^{-6}\right.$ au lieu de $\delta_{\mathrm{m}}=8,50 \times 10^{-6}$ pour $\mathrm{CuK}_{\alpha_{1}}$ l et d'une importante zone de densification sous-jacente 


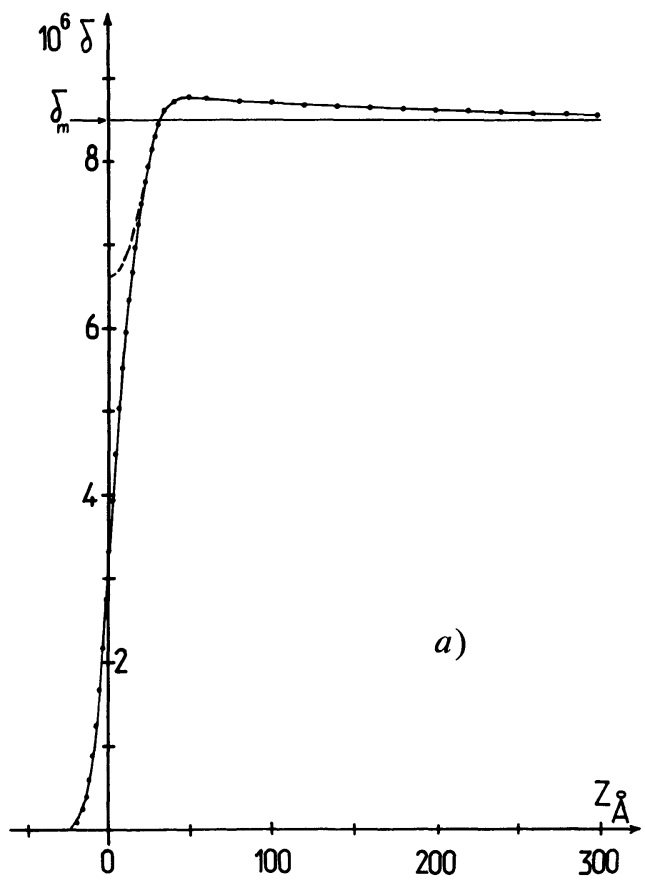

Fig. 7. - a) Profil $\delta(Z)$ d'un verre alumino-silicaté poli à l'oxyde de fer sur poix de Bourgogne jaune. On notera la similitude de forme entre ce graphe et celui de la silice pure présenté en $4 b . b$ ) Evolution $\mathrm{du}$ profil précédent en fonction du traitement thermique. Les graphes de symbole $(O \circ \mathrm{O})$ et $(\Delta \Delta \Delta)$ correspondent à un traitement d'une heure à $400^{\circ} \mathrm{C}$ et $600{ }^{\circ} \mathrm{C}$ respectivement. On constate ainsi qu'après l'élimination de la densification, les caractéristiques de la couche limite superficielle (indice, épaisseur) restent sensiblement les mêmes (- - - ), seule la rugosité de surface étant légèrement abaissée.

$\left(\tau_{1}=200 \AA\right)$. Le maximum de densité se situe à $48 \AA$ sous la surface moyenne et vaut 2,71

$$
\left(\delta_{\max }=8,77 \times 10^{-6}\right) \text {. }
$$

La rugosité de surface de l'échantillon présenté ici est de $9 \AA$.

La courbe en pointillés de la figure $7 b$ concerne le même échantillon porté pendant une heure et sous vide à $400^{\circ} \mathrm{C}$. On constate là aussi, que si le traitement thermique a fait disparaître la densification sousjacente, il subsiste toujours une pellicule limite, de densité inférieure à la masse. L'indice de surface augmente légèrement $\left[\delta_{0}=7,15 \times 10^{-6}\right]$, alors que l'épaisseur moyenne et la rugosité diminuent

$$
\left[\tau_{2}=19 \AA \text { et } \sigma=7,95 \AA\right] .
$$

Les traitements supplémentaires effectués à $500^{\circ} \mathrm{C}$ puis à $600{ }^{\circ} \mathrm{C}$ n'apportent qu'une nouvelle diminution de la rugosité de surface, l'allure du graphe $\delta(Z)$ restant sensiblement la même. Ainsi, la courbe en

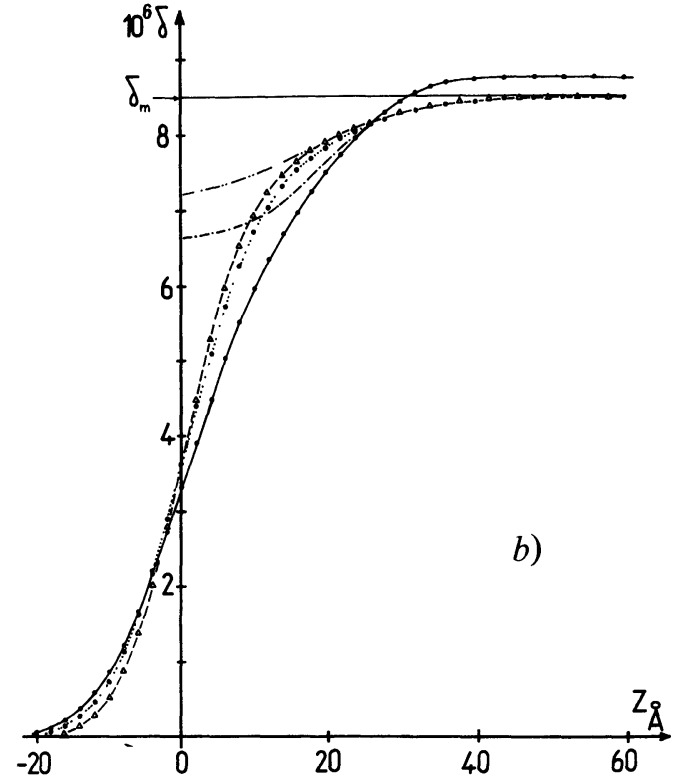

[a) E.D.P. of an alumino-silicate glass polished with ferrous oxide on gas pitch. Notice the similarity with the E.D.P. of silica glass (Fig. 4b). b) E.D.P. dependence on heat treatment. Curves designated by $(O \circ O)$ and $(\Delta \Delta \Delta)$ refer to polished sample after heat treatment at respectively 400 and $600^{\circ} \mathrm{C}$. When the compaction of the sublayer is vanishing we find again that the outer layer is nearly inchanged, although the surface roughness decreases slightly.]

tirets de la figure $7 b$, relative au dernier traitement $\left(600{ }^{\circ} \mathrm{C}\right.$ pendant une heure), correspond aux valeurs paramétriques suivantes :

$$
\delta_{0}=7,05 \times 10^{-6}, \quad \tau_{2}=17 \AA, \sigma=6,85 \AA .
$$

3.3 Borosilicate. - Nous avons examiné deux types de verres borosilicatés : le borosilicate Crown B 1664 (Sovirel-Parra-Mantois) et le Pyrex 7740 (Corning) dont on trouvera ci-dessous les compositions pondérales approximatives et les valeurs d'indice massique pour le rayonnement $\mathrm{CuK}_{\alpha_{1}}$ (Tableau I).

3.3.1 Borosilicate Crown B 1664. - Les graphes $\delta(Z)$ de la figure 8 concernent un polissage machine effectué sur poix de Norvège noire à l'aide d'une suspension aqueuse d'oxyde de cérium (cérox 1670 Rhône Poulenc), d'oxyde de fer (309 rouge-Goldring) ou d'oxyde d'étain (potée d'étain $33 \% \mathrm{SnO}_{2}$ Rhône Poulenc). D'une façon générale on n'observe plus de densification superficielle, mais au contraire une

Tableau I.

\begin{tabular}{lccccccccc}
\multicolumn{1}{c}{ Verre } & $\rho \mathrm{g} / \mathrm{cm}^{3}$ & $10^{6} \delta_{\mathrm{m}}$ & $10^{6} \beta_{\mathrm{m}}$ & $\mathrm{SiO}_{2}$ & $\mathrm{~B}_{2} \mathrm{O}_{3}$ & $\mathrm{Na}_{2} \mathrm{O}$ & $\mathrm{K}_{2} \mathrm{O}$ & $\mathrm{BaO}$ & $\mathrm{Al}_{2} \mathrm{O}_{3}$ \\
$\quad-$ & - & - & - & - & - & - & - & - & - \\
B 1664 & 2,52 & 8,07 & 0,136 & 67,5 & 12 & 9 & 9,5 & 2 & \\
Pyrex 7740 & 2,23 & 7,186 & 0,087 & 81 & 13 & 3 & 1 & & 2
\end{tabular}




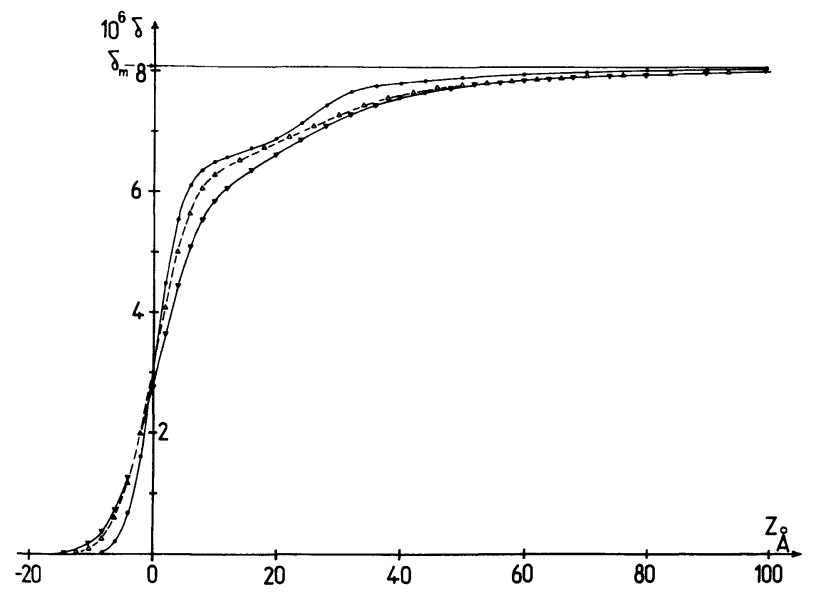

Fig. 8. - Modification du profil $\delta(Z)$ du borosilicate crown (B 1664) poli sur poix de Norvège noire, en fonction de la nature de la potée de polissage : oxyde de cérium $(\nabla \nabla \nabla)$, oxyde de fer $(\Delta \Delta \Delta)$ ou oxyde d'étain $(\bullet \bullet \bullet)$.

[E.D.P. of a borosilicate crown glass polished on swedish pitch with various oxides : cerium $(\boldsymbol{\nabla} \nabla)$ iron $(\Delta \Delta \Delta)$ or tin $(\bullet \bullet \bullet)$.

diminution de la compacité par rapport à la masse ceci sur une profondeur de $100 \AA$ environ. Ce défaut de compacité diminue nettement lorsqu'on passe de l'oxyde de cérium à l'oxyde de fer puis à la potée d'étain (les variations d'indice intégrées $J$ se chiffrent respectivement à $-72,6 \times 10^{-6}-64,25 \times 10^{-6}$ et $\left.-53,82 \times 10^{-6}\right)$.

Il en est de même pour la rugosité $(5,4 \AA, 4,65 \AA$ et $3,3 \AA$ respectivement). On constate en outre, comme précédemment pour la silice pure et l'alumino-silicate, la présence d'une couche superficielle limite d'épaisseur très faible $\left(\tau_{2}\right.$ prend ici les valeurs respectives 23,25 et $26 \AA$ ). Notons que l'allure du graphe $\delta(Z)$ ne permet de déceler sa présence que dans la mesure où, d'une part, la variation d'indice dans cette pellicule reste faible et, d'autre part, les rugosités des deux interfaces limites sont suffisamment faibles, conditions qui ne semblent satisfaites que pour le graphe relatif à $\mathrm{SnO}_{2}$. L'indice de surface $\delta_{0}$ est sensiblement le même pour ces trois polissages $\left(5,5 \times 10^{-6}\right.$ et $\left.6 \times 10^{-6}\right)$. A titre de comparaison, rappelons (L. Névot [32], page V 38) les valeurs des divers paramètres qui permettent de simuler la courbe expérimentale présentée sur la figure 1 et relative à un verre BSC-B 1664 poli manuellement à l'oxyde de cérium sur poix de Bourgogne jaune :

- rugosité de surface : $8,3 \AA$,

- indice en surface : $\delta_{0}=5,7 \times 10^{-6}$,

- épaisseur de la couche superficielle limite : $\tau_{2}=19 \AA$,

- $J=-92,4 \times 10^{-6}$.

L'allure générale du graphe $\delta(Z)$ correspondant à ces valeurs est tout à fait semblable à celle du graphe homologue de la figure 8 .

Les divers oxydes utilisés n'ayant pas le même mordant, c'est-à-dire la même efficacité de polissage, il est nécessaire de polir plus ou moins longtemps pour obtenir la même qualité de poli, autrement dit le même état de surface tel qu'on peut l'observer à l'aide d'un microscope optique. Il était donc important de déterminer l'influence éventuelle de la durée de polissage sur la valeur des divers paramètres du profil $\delta(Z)$.

$\mathrm{Au}$ cours d'un polissage machine à l'oxyde de cérium et sur poix de Norvège, nous avons prélevé des échantillons à intervalles de temps réguliers, le premier après une demi-heure seulement et le dernier après douze heures de polissage. L'examen des valeurs absolues des flux réfléchis lorsque $\theta$ est inférieur à $\theta_{c}$, révèle que l'on se rapproche progressivement de la valeur du flux enregistré dans le faisceau incident, l'amélioration étant très nette jusqu'à quatre heures de polissage, puis relativement faible ensuite. L'analyse des surfaces par microscopie optique indique la même évolution pour ce qui est de l'élimination des défauts de surface tels que les piqûres et les crevasses de toute sorte. Les valeurs absolues des flux réfléchis à incidence fixe pour $\theta$ supérieur à $\theta_{\mathrm{c}}$, augmentent elles aussi de la même façon. Qui plus est, une fois normalisées sur la valeur maximum du flux réfléchi pour $\theta<\theta_{\mathrm{c}}$, on constate que les diverses courbes de réflexion spéculaire se superposent presque exactement, et par suite conduisent au même graphe $\delta(Z)$, sauf pour celle relative à une demi-heure de polissage où la limite de réflexion totale apparaît légèrement décalée vers les angles plus petits. La rugosité, entre autres, ne paraît donc pas liée à la durée de polissage, du moins pour ce qui est de la contribution des fréquences spatiales assez élevées $\left(>0,03 \mu \mathrm{m}^{-1}\right)$. La diffraction engendrée par les fréquences spatiales inférieures à cette valeur est recueillie par la fente du compteur et ne peut donc être séparée de la réflexion spéculaire (voir L. Névot [32], Chap. IV-2).

On peut donc concevoir qu'en augmentant la durée de polissage on ne fait qu'accroître la surface moyenne des plateaux (lesquels jouent le rôle de bons réflecteurs pour les rayons $X$, d'où une augmentation du flux réfléchi) sans en modifier la granulométrie, cette dernière étant probablement atteinte dès les premières minutes de polissage et caractéristique de l'interaction à la fois mécanique et chimique entre la potée de polissage et le verre.

Le fait que le graphe $\delta(Z)$ ne dépende pratiquement pas de la durée de polissage pour le B 1644 est à rapprocher des observations de Sakata [17] par ellipsométrie, sur un flint dense poli sur poix, à l'oxyde de cérium. L'auteur constate que la variation d'indice moyen de la couche superficielle par rapport à la masse reste la même pour une durée de polissage comprise entre une demi-heure et trois heures. De plus l'épaisseur moyenne de cette couche n'augmente pas suffisamment, compte tenu des incertitudes de ces mesures ellipsométriques, pour que l'on puisse conclure à une évolution significative [voir Fig. $3 b$, page 176]. 
Comme nous l'avons souligné dans le chapitre I, la variation de $\delta(Z)$ est essentiellement le reflet du changement de compacité, attribuable d'une part à l'effet de rugosité lequel prédomine pour le voisinage immédiat du plan moyen de la surface, et d'autre part, pour le matériau sous-jacent, soit à une densification, soit à une lacunisation (microporosité), laquelle peut se caractériser par la variation d'indice intégrée $J$. Dans le cas du B 1664, étant donnée la teneur assez élevée en ions alcalins $\mathrm{Na}, \mathrm{K}$, la densification permanente que l'on pourrait atteindre sous la seule action des forces de pression exercées par les poudres de polissage, est certainement très faible si l'on en croit les expériences de Bridgman et Simon [33]. La valeur de $J$ est donc ici étroitement liée au seul processus de lacunisation. La formation de pores résulte de la diffusion vers la surface des ions métalliques (diffusion favorisée par l'élévation de la température dans la zone de friction) et de leur extraction en raison de l'action lixiviante de l'eau. Ainsi la comparaison des spectres d'électrons Auger d'une surface polie et d'une surface de fracture (ou cassure) révèle pour les premiers l'absence des pics du sodium et du bore, ainsi que la forte atténuation de celui du potassium lorsque le polissage a été effectué avec les oxydes de cérium ou de fer. On notera que le sodium n'apparaît pas non plus sur les seconds, ceci vraisemblablement en raison de sa très grande mobilité sous l'influence du champ électrique local, lequel est inhérent à la pénétration des ions incidents. Pour ralentir la migration du sodium il apparaît nécessaire de refroidir fortement l'échantillon (Pantano et al. [40]). Le spectre Auger relatif à la surface polie à la potée d'étain, se distingue des précédents par un large pic de bore, quoique légèrement moins intense que celui relatif à la composition massique.

Soulignons également l'absence de toute raie du calcium, du cérium, du fer et de l'étain, ce qui confirme à la fois la qualité du nettoyage, et la non-implantation de ces différents ions au cours du polissage.

3.3.2 Pyrex 7740. - Le cas du Pyrex est particulièrement intéressant car sa teneur en ions alcalins étant très faible, on peut espérer mettre en évidence l'effet concurrentiel de la densification et de la lacunisation. Le polissage à la potée d'étain étant nettement plus doux que celui au cérium, le graphe $\delta(Z)$ relatif à ce polissage devrait faire ressortir plus nettement le processus de densification. C'est effectivement ce qui est observé sur les graphes de la figure 9 . La variation d'indice intégrée $J$ est égale à $+14,1 \times 10^{-6}$ dans le cas de $\mathrm{SnO}_{2}$ et à $-13,9 \times 10^{-6}$ pour l'oxyde de cérium : l'effet relatif des deux processus est donc inversé sur cet exemple. Dans le premier cas la valeur maximum de $\delta$ est de $7,35 \times 10^{-6}$ pour la cote $+41 \AA$ et dans le second, elle n'est plus que de 7,21 $\times 10^{-6}$ pour la cote $+37 \AA$.

Par ailleurs on retrouve le même indice de surface $\delta_{0}=7,0 \times 10^{-6}$. Enfin, on constate, comme précédemment pour le B 1664 , que la rugosité est légè-

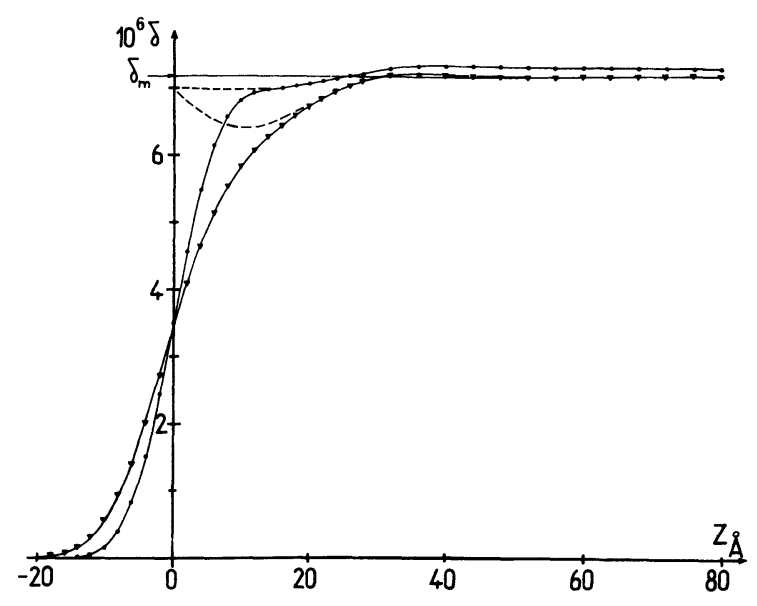

Fig. 9. - Profils $\delta(Z)$ pour le Pyrex 7740 poli sur poix de Norvège noire à l'oxyde de cérium $(\boldsymbol{\nabla} \nabla \nabla)$ ou à la potée d'étain $(\bullet \bullet \bullet)$. Les tracés en tirets concernent l'expression $\left(9^{*}\right)$, et font donc abstraction des rugosités de surface. On remarque ainsi plus directement que la lacunisation au voisinage immédiat de la surface est nettement plus importante dans le cas du polissage au cérox.

[E.D.P. of pyrex glass polished on swedish pitch with either cerium oxide $(\boldsymbol{\nabla} \nabla \boldsymbol{\nabla})$ or tin oxide $(\bullet \bullet \bullet)$. Dashed lines agree with expression $\left(9^{*}\right)$ and therefore don't take into account the surface roughness. Thus the depletion process near the surface clearly appears to be more accentuated when using cerium oxide.]

rement plus faible avec $\mathrm{SnO}_{2}(5,1 \AA$ au lieu de $7,1 \AA)$ et que l'épaisseur $\tau_{2}$ de la couche limite est au contraire légèrement supérieure $(25 \AA$ au lieu de $19 \AA)$.

4. Conclusion. - L'analyse, par réflexion rasante de rayons $\mathbf{X}$, des surfaces de verres silicatés, polis mécaniquement sur poix à l'aide de suspensions aqueuses d'oxydes de cérium, de fer ou d'étain, révèle qu'il se forme au cours du polissage une couche superficielle de compacité et (ou) de composition différentes de celles de la masse. Cette couche est, en fait, composée de deux zones bien distinctes. La première, située au voisinage immédiat de la surface, ne concerne que quelques dizaines d'angströms tout au plus, et se caractérise par une densité inférieure à celle de la masse $(9 \%$ pour la silice pure, $16 \%$ pour le verre alumino-silicaté et $30 \%$ environ pour le verre B 1664) ce qui semble conforter l'hypothèse de l'hydrolyse. Toutefois l'examen des valeurs de $\tau_{2}$ pour les polissages effectués dans les mêmes conditions, de poix et d'oxyde notamment, révèle que celles du pyrex, de l'aluminosilicate et du B 1664, sont assez semblables alors que leurs compositions sont quelque peu différentes. Par contre leurs microduretés Knoop paraissent assez proches (valeur limite de 450 à $495 \mathrm{~kg} / \mathrm{mm}^{2}$ [41]). Dans le même ordre d'idée, l'épaisseur $\tau_{2}$ pour la silice pure est légèrement plus faible, mais sa microdureté est aussi plus élevée (microdureté Knoop de $600 \mathrm{~kg} / \mathrm{mm}^{2}$ pour une charge de $100 \mathrm{~g}$ ). Il y aurait donc une certaine relation entre l'épaisseur de la couche superficielle limite et la microdureté du verre. A cet égard nous avons analysé deux verres de composition totalement différentes, un flint 
extra dense (D 8825, Sovirel Parra-Mantois) et un aluminophosphate, nettement plus mous que les précédents, mais de même microdureté (microdureté Vickers de 325 et $315 \mathrm{~kg} / \mathrm{mm}^{2}$ respectivement, pour une charge de $50 \mathrm{~g}$, M. Prod'homme [42]). Les valeurs obtenues pour $\tau_{2}$ ont été de 33 et $34 \AA \AA$ respectivement et les variations de densité en surface par rapport à la masse de $-35 \%$ dans les deux cas.

Est-ce à dire que le polissage de la surface s'effectue par simple abrasion mécanique et que la couche superficielle limite est à la fois plus ou moins épaisse et poreuse, selon la dureté du verre surfacé. En fait, nous avons tout lieu de penser que cette pellicule se comporte plutôt comme une pâte à la fois homogène et compacte (bien que de densité inférieure à la valeur massique) et non pas comme un gruyère plus ou moins poreux.

Ainsi lorsqu'on dépose sur un substrat de verre poli un film mince, d'or par exemple, il ne se produit pas de diffusion des atomes d'or dans le verre. En effet l'analyse par électrons Auger de la surface du substrat, une fois le film d'or retiré (à l'aide d'un simple ruban adhésif par exemple), ne révèle aucune trace d'or. De même, la surface d'or en contact avec le verre ne présente aucune trace de silicium. Une autre confirmation est fournie par l'étude en réflexion rasante de rayons $\mathrm{X}$ de ces films d'or (d'épaisseur égale à quelques centaines d'angströms) sur substrat de verre poli : on retrouve pour l'interface or-verre la même valeur de rugosité que celle obtenue par analyse directe du verre nu. Par conséquent, contrairement aux conclusions de H. Sakata [17], il nous semble que ces divers résultats vont dans le sens d'un fluage plastique superficiel, ce qui n'interdit pas, loin s'en faut, la possibilité de réactions chimiques, en particulier l'hydrolyse, avec la potée de polissage. Précisons aussi que dans nos conditions de polissage, soit sous faible pression nominale, ce fluage ne provoque pas d'enrobage, ou incrustation, des poudres à polir, ce que n'auraient pas manqué de déceler les analyses par électrons Auger.

Sous cette mince pellicule hydrolysée se présente une zone assez épaisse (de une à quelques centaines d'angströms) qui, selon les cas, est soit plus compacte (densification) soit au contraire moins compacte (lacunisation) que la masse de l'échantillon. Dans ces deux cas la variation de la compacité avec la profondeur est décrite par une simple loi exponentielle.

Ce phénomène de densification n'est observé que sur les verres dont la composition est exempte d'oxydes alcalins ou n'en contient que très peu (quelques \% $\mathrm{au}$ plus). Dans nos conditions de polissage, où la pression nominale est de l'ordre de quelque $10^{2} \mathrm{Nm}^{-2}$, la densification maximale ne dépasse pas $3 \%$ environ, et peut être éliminée en portant l'échantillon à $400^{\circ} \mathrm{C}$ pendant une heure environ. Ceci laisse supposer que la zone densifiée est soumise à des contraintes résiduelles, contraintes développées vraisemblablement au cours du polissage (et non pas pendant le douci) à la fois par le cisaillement et les fortes pressions exercées localement par les grains d'oxyde.

Dans le cas des silicates à teneur assez élevée en oxydes alcalins ( $20 \%$ environ) c'est au contraire un défaut de compacité (microporosité ou lacunisation) qui caractérise la zone sous-jacente. Une telle lacunisation ne peut être attribuée qu'à la migration (ou diffusion) des ions $\mathrm{Na}^{+}$et (ou) $\mathrm{K}^{+}$vers la surface et leur extraction en raison de l'action lixiviante de l'eau (incorporée dans la potée de polissage). Il est d'ailleurs assez significatif de constater que la valeur de $\tau_{1}$ est nettement plus élevée pour les verres plus sensibles à l'humidité ambiante. D'autre part lorsqu'on compare les polissages machine effectués avec différents oxydes, en suspension aqueuse telle que la dilution soit sensiblement la même, on constate que non seulement la valeur de $\tau_{1}$ reste pratiquement constante mais que la contribution afférente à $\tau_{1}$ dans $J$, c'est-à-dire dans le défaut global de densité, l'est aussi.

Les modifications inhérentes à l'oxyde utilisé comme ingrédient de polissage concernent principalement la rugosité de surface et, dans une moindre mesure, la composition superficielle. Dans l'ensemble elles paraissent liées à l'efficacité polissante de la potée. Ainsi, la rugosité de surface du $\mathrm{B} 1664$ diminue de $\mathrm{CeO}_{2}$ à $\mathrm{Fe}_{2} \mathrm{O}_{3}$ puis $\mathrm{SnO}_{2}$ et peut être abaissée ainsi à $3 \AA$ environ.

Nous venons d'étudier plus particulièrement les surfaces de verres silicatés polis non seulement parce que le polissage du verre constitue un processus intéressant en soi mais aussi parce que le verre est fréquemment utilisé comme substrat pour l'analyse des matériaux déposés sous la forme de films minces et qu'une étude précise de ces films passe d'abord par une caractérisation fine du substrat. Mais, ainsi que nous l'avons signalé à plusieurs reprises, la technique d'analyse par réflexion rasante de rayons $X$ convient également à toute surface de matériau, amorphe ou cristallisé, pourvu que sa planéité soit suffisante sur une aire minimum de l'ordre de $1 \mathrm{~cm}^{2}$. Seront ainsi déterminés, de manière non destructive, à la fois la rugosité de la surface et le profil de la densité électronique moyenne en fonction de la profondeur. Les conditions restrictives d'utilisation de notre modèle théorique sont d'une part, une distribution gaussienne des rugosités de surface et d'autre part une certaine stratification de la densité électronique dans la couche de passage, ou interface, entre la surface et le cœur du matériau. Aussi toute contamination ou attaque, naturelle ou provoquée, de la surface devra-t-elle s'effectuer de manière suffisamment régulière, pour entrer dans le cadre de cette théorie.

Remerciements. - Nous tenons à exprimer notre reconnaissance à la Société Corning, et plus particulièrement à M. G. Guillevic, pour la fourniture gracieuse des échantillons de pyrex et de verre alumino- 
silicaté, et la communication de leurs caractéristiques techniques.

Nous remercions également M. J. P. Chauvineau pour ses analyses de surface par électrons Auger.

Le polissage des échantillons a été effectué dans les ateliers de l'Institut d'Optique sous la direction de MM. J. P. Marioge et G. Clinard, ainsi que par M. L. Constanciel. Le nettoyage méticuleux des surfaces a été confié à Mme Valiergue.

\section{Bibliographie}

[1] Rayleigh, Nature 64 (1901) 385.

[2] BeIlby, G. T., Proc. R. Soc. A-72 (1903) 218-226.

[3] Klemm, W., SmeKal, A. G., Naturwissenschaften 29 (1941) 688-769.

[4] BRÜChe, E., POPPA, H., Glastechn Ber. 29 (1956) 183-192.

[5] GREBENŠČIKOV, I., Keram. Steklo 7 (1931) 36.

GreBENŠČIKOV, I., Sot. Reconstr. Nauka 2 (1935) 22.

[6] Kaller, A., Silikaty 7 (1956) 380-390.

[7] BourgeauX, M., Peyches, I., Colloque sur la nature des surfaces vitreuses polies, Paris (1959) publié par U.S.C.V. Charleroi-Belgique (1960) 95-120.

[8] BenNett, J. M., Appl. Opt. 15 (1976) 2705-2721.

[9] Rynd, J. P., Rastogi, A. K., Surf. Sci. 48 (1975) 22-43.

[10] Onoda, G., Dove, D., Pantano, C., Mater. Sci. Res., Vol. 7 (1974) 39-56.

[11] HEYNDRYCKX, P., Glastechn Ber. 44 (1971) 543-547.

[12] Pantano, C., Dove, D., Onoda, G., Appl. Phys. Lett. 26 (1975) 601-602.

[13] Croce, P., Névot, L., Revue Phys. Appl. 11 (1976) 113-125.

[14] Legrand, C., Merx, H., Navez, M., Sili. Ind. 27 (1962) 483-488.

[15] Yokota, H., Kinosita, K., Sakata, H., Japan J. Appl. Phys. 3 (1964) 805-806.

[16] Yokota, H., Sakata, H., Nishibori, M., Kinosita, K., Surf. Sci. 16 (1969) 265-274.

[17] Sakata, H., Japan J. Appl. Phys. 12 (1973) 173-181.

[18] Croce, P., Prod'homme, L., Nouv. Rev. Opt. 7 (1976) 121-132.

[19] Rayleigh, Proc. R. Soc., A-79 (1907) 399-416.

[20] Petzold, W., Z. Angew. Phys. 15 (1963) 525-532.
[21] Cromer, D. T., Liberman, D., J. Chem. Phys. 53 (1970) 1891-1898.

[22] Hink, W., Petzold, W., Z. Angew. Math. Physik 10 (1968) 553-562.

[23] Kunnen, G., Optik 26 (1967-1968) 582-593.

[24] Croce, P., Névot, L., Pardo, B., C. R. Hebd. Séan. Acad. Sci. B-274 (1972) 803-806, 855-858.

[25] Croce, P., Prod'homme, L., Revue Phys. Appl. 12 (1977) 1641-1648.

[26] Croce, P., Nouv. Rev. Opt. 8 (1977) 127-137.

[27] Croce, P., Rev. Phys. Appl. 12 (1977) 87-91.

[28] Croce, P., Nouv. Rev. Opt. 9 (1978) 61-63.

[29] Névot, L., Croce, P., J. Appl. Crystallogr. 8 (1975) 304-314.

[30] Croce, P., Névot, L., J. Appl. Crystallogr. 7 (1974) 125-130.

[31] Devant, G., Thèse Orsay (1969) no A-453.

[32] NÉvot, L., Thèse Orsay (1978) $\mathrm{n}^{\circ} 1954$.

[33] Bridgman, P. W., Šimon, I., J. Appl. Phys. 24 (1953) 405-413.

[34] Mackenzie, J. D., J. Am. Ceram. Soc. 46 (1963) 461-470 et 470-476.

[35] Arndt, J., Stöffler, D., Phys. Chem. Glasses 10 (1969) $117-124$

[36] OTTE, G., J. Sci. Instrum. 42 (1965) 911-912.

[37] OtTE, G., J. Sci. Instrum. (J. of Physics E) 2 (1969) 622-623.

[38] Mozzi, R. L., Warren, B. E., J. Appl. Crystallogr. 2 (1969) 164-172.

[39] Couty, R., Thèse Paris VI (1977).

[40] Pantano, C., Dove, D., Onoda, G., J. Vac. Sci. Technol. 13 (1976) 414-418.

[41] Guillevic, G., Communication privée.

[42] Prod'homme, M., Phys. Chem. Glasses 9 (1968) 101-105. 